\title{
Optimization of culture conditions by response surface methodology and unstructured kinetic modeling for bioactive metabolite production by Nocardiopsis litoralis VSM-8
}

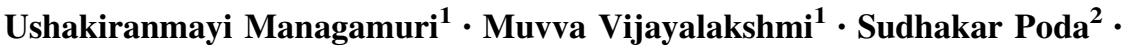 \\ V. S. Rama Krishna Ganduri ${ }^{2,3}$ - R. Satish Babu ${ }^{4}$
}

Received: 23 July 2016/Accepted: 27 September 2016/Published online: 8 October 2016

(C) The Author(s) 2016. This article is published with open access at Springerlink.com

\begin{abstract}
Response surface methodology-based central composite design on five variables incubation time, $\mathrm{pH}$, temperature, sucrose concentration, and soya peptone concentration was employed for optimization of the production of bioactive compounds by Nocardiopsis litoralis strain VSM 8. The main quadratic effects and interactions of the five variables on the production of bioactive metabolites were investigated. A second-order polynomial model produced a satisfied fit for experimental data with regard to the production of the bioactive metabolites. Regression analysis showed that high $R^{2}$ values of all the five responses are significant and adjusted $R^{2}$ values showed good agreement with the experimental and predicted values. The present model was used to evaluate the direct interaction and quadratic effects to optimize the physico-chemical parameters for the production of bioactive metabolites that inhibit the pathogenic microorganisms measured in terms of zones of inhibition (responses). Mathematical kinetic model development and estimation of kinetic parameters also showed good approximation in terms of model fitting and regression analysis.
\end{abstract}

Muvva Vijayalakshmi

profmv108@gmail.com

1 Department of Botany and Microbiology, Acharya Nagarjuna University, Nagarjuna Nagar, Guntur 52510, Andhra Pradesh, India

2 Department of Biotechnology, Acharya Nagarjuna University, Nagarjuna Nagar, Guntur 52510, Andhra Pradesh, India

3 Department of Biotechnology, K L University, Vaddeswaram, Guntur, Andhra Pradesh, India

4 Department of Biotechnology, National Institute of Technology, Warangal, Telangana, India
Keywords Optimization - Bioactive metabolites · RSM · CCD $\cdot$ Kinetic modeling

\section{Introduction}

Microorganisms dwelling in extreme environments are prolific producers of several bioactive compounds that have evolved due to adaptation of the extreme environmental conditions in terms of metabolic biochemistry. Actinomycetes are well documented for their capacity to produce bioactive metabolites with complex diversity and diverse biological activities. The genes involved in the synthesis of secondary metabolites remain undiscovered due to absence of transcription under conventional laboratory conditions, but mathematical models predict thousands of unexplored secondary metabolites from actinomycete genomes. Actinomycetes are stable, persistent, and active component of the marine microbial communities. Discovery of novel actinobacteria, which operate unusual metabolic pathways, has the ability to produce complex chemical compounds with potential biological activities.

Optimization of physiological parameters and the supplementary nutrition for the biosynthesis of bioactive metabolites can be achieved by the systemic study. The important parameters such as cultural conditions and media constituents are the significant factors influencing the high yield of antimicrobial compounds. Often the production conditions are very similar among the closely related actinomycetes, and hence, optimization of growth and production conditions is very crucial for the maximum production of potential bioactive compounds (Pfefferle et al. 2000; Kiranmayi et al. 2011). Media optimization explores a sequence of phases with specific set of optimal conditions fixed by different methodologies (Shekar et al. 2014). 
Traditional one-factor-at-time (OFAT) optimization is laborious and time-consuming, and also misleading conclusions may be drawn, because individual factor interactions are ignored. Hence, establishing the optimized conditions is cumbersome, and therefore, cultural conditions need to be optimized to maximize the production of bioactive compounds with preserved biological activities (Ilaiyaraja et al. 2015). Statistical optimization approach is advantageous than to the traditional practice of changing one variable at a time (Collaa et al. 2016). Response surface methodology (RSM) quantifies the relationship between the controllable input parameters and obtained response. RSM is a powerful statistical experimental approach used in mathematical modeling, and an ideal process for variable standardizing strategy for optimization of the target metabolite production and simultaneously evaluates the relative significance and interactive effects among different variables (Souagui et al. 2015). Central composite design (CCD) is a program of RSM that is embedded with factorial or fractional factorial design with center points that are augmented with a group of "star points" that allows estimation of curvature.

Furthermore, the successful design and operation of fermentation process, in which biochemical transformation occurs in controlled conditions, need careful understanding of complex metabolic reactions. This could be supported by mathematical modeling that describes the process simpler with good representation. Fermentation kinetic models allow the bioengineer to get insight and deep knowledge on the mechanism of synthesis of bioactive metabolites for its yield and productivity from fermentation studies. Furthermore, the evaluation of assumed unstructured models with the experimental data for comparison to find the best model that describes the system. In general, unstructured models consider the cell mass as a whole to explain the biological system and are more effective in elucidating the fermentation profiles of microbial process as for bio products (Rajendran and Thangavelu 2008; Rama Krishna et al. 2016).

Hence, the objectives of this study include the statistical optimization of process parameters for bioactive metabolite production using RSM and to estimate the kinetic parameters of actinomycetes fermentation using $N$. litoralis strain VSM 8 (KT901293).

\section{Materials and methods}

\section{Isolation and identification of actinomycetes}

As a part of our ongoing research on bioactive metabolites, one promising strain with good antimicrobial potential was identified as $N$. litoralis strain VSM 8, isolated from deep sea sediment samples of Bay of Bengal, Visakhapatnam using soil dilution plate technique on actinomycetes isolation agar medium containing 2 -g sodium caseinate, $0.1-\mathrm{g}$ L-asparagine, 4-g sodium propionate, 0.5-g dipotassium phosphate, 0.1-g magnesium sulfate, 0.001-g ferrous sulfate, and $5 \mathrm{ml}$ glycerol per $1000 \mathrm{ml}$ distilled water $(\mathrm{pH} 8)$. The strain has been deposited in NCBI Genbank with an accession number KT901293. The strain was maintained on Yeast extract Malt Extract Dextrose (YMD) agar medium at $4{ }^{\circ} \mathrm{C}$. An attempt was made in the study to optimize the culture conditions for enhancing the production of bioactive metabolites by the strain using RSM.

\section{Optimization of screened process parameters for bioactive metabolite production by $N$. litoralis strain VSM 8}

RSM applies the statistical and mathematical approach for modeling, designing, and analyzing the engineering problems. It is used to optimize the response surface that influences the various parameters and relationship between the input parameters and the obtained response quantitatively (Parmjit 2008). Relationship between response and independent variables is unknown. Therefore, it is important to execute RSM to find the legitimate practical relation between the responses and the set of independent variables. In this study, the use of RSM to determine the optimum conditions of $N$. litoralis strain VSM 8 for the bioactive metabolite production under a wide range of physical conditions was performed.

To design minimum number of experimental runs, a full factorial central composite face-centered design (CCFD) with five independent variables and their combinations was used to optimize the response with the region of threedimensional observation spaces. Design Expert software (Version 7.0 State-Ease, Inc., USA) was used to design the experiments for bioactive metabolites production. Using CCD of RSM, the most significant variables (A, B, C, D, and $\mathrm{E}$ ) at their optimum levels were identified for maximal response in terms of antimicrobial activity of bioactive metabolites measured as zone of inhibition. Five independent variables selected in this study include A-Incubation time, B-pH, C-Temperature, D-Sucrose, and E-Soya peptone. A total of 50 experiments were obtained using following equation that have $2^{5}$ full factorial CCD for five variables comprising 32 factorial points, 10 axial points, and 8 replicates:

$N=2^{n}+2 n+n_{\mathrm{c}}=2^{5}+2 \times 5+8=50$,

where $N$ is total number of experimental runs to be performed, $n$ is number of variables (factors), and $n_{\mathrm{c}}$ is number of replicates at center points.

The central coded value of all the variables was considered as zero. Low and high ranges of all the variables 
Table 1 Experimental range of factors studied using CCD in terms of coded and actual factors

\begin{tabular}{lllll}
\hline Factors & Symbols & \multicolumn{3}{l}{ Actual levels of coded factors } \\
\cline { 3 - 5 } & & -1 (low) & 0 (middle) & +1 (high) \\
\hline Time of incubation (days) & $A$ & 10 & 11 & 12 \\
$\mathrm{pH}$ & $B$ & 7 & 8 & 9 \\
Temperature $\left({ }^{\circ} \mathrm{C}\right)$ & $C$ & 25 & 30 & 35 \\
Sucrose concentration $(\% \mathrm{w} / \mathrm{v})$ & $D$ & 1 & 2 & 3 \\
Soya peptone concentration $(\% \mathrm{w} / \mathrm{v})$ & $E$ & 0.5 & 1.0 & 1.5 \\
\hline
\end{tabular}

used in RSM and the complete experimental plan with values in actual and coded form are presented in Table 1.

\section{Statistical analysis}

The model was statistically analyzed to evaluate the analysis of variance (ANOVA). To analyze the fit and prediction accuracy of the model constructed, correlation coefficients $\left(R^{2}\right)$, adjusted determination coefficient (Adjusted- $R^{2}$ ), root mean square error (RMSE), and absolute average deviation (AAD) were carried out between experimental and predicted data. The data obtained were subjected to graphical and regression analysis using Design Expert software. The experimental errors and reproducibility of the data were determined by the central points. To minimize the effect of the uncontrolled factors, the experimental sequence was randomized. The quadratic regression equation was used with each variable to develop an empirical model which correlated the response (bioactive metabolite production) to five variables, as per the following equation (Cui et al. 2016):

$Y=\beta_{0}+\sum_{i=1}^{n} \beta_{i} X_{i}+\left(\sum_{i=1}^{n} \beta_{i i} X_{i}\right)^{2}+\sum_{i=1}^{n-1} \sum_{j=i+1}^{n} \beta_{i j} X_{i} X_{j}$,

where $Y$ is predicted response, $\beta_{0}$ is intercept coefficient, $\beta_{i}$ is linear coefficient, $\beta_{i j}$ is interaction coefficients, $\beta_{i i}$ is quadratic coefficients, and $X_{i}$ and $X_{j}$ are coded values of the five additive variables.

\section{Unstructured kinetic modeling of $N$. litoralis strain VSM 8}

The growth of halophilic marine actinomycete with limiting carbon substrates influences the bioactive metabolite production. Basic mathematical and unstructured kinetic models quantitatively describe the substrate utilization and growth-associated production formation kinetics in a batch system, and the similar equations were also developed by many researchers (Mohammad et al. 1995; Cheng et al. 2010; Li et al. 2015). Models of logistic and LuedekingPiret were used to simulate the cell growth and bioactive metabolite production of $N$. litoralis strain VSM 8 (KT901293). The data acquired from the models were used to calculate the specific cell growth rate $\left(\mu_{\max }\right)$, day $^{-1}$, specific production rate of bioactive metabolite, day ${ }^{-1}$.

Under optimal growth conditions and no effects of substrate and product inhibition, growth kinetic model of $N$. litoralis strain VSM 8 (KT901293) (X) (as per Malthus's law), in a batch fermentation, is best described as logistic function (Leroy and de Vuyst 1999):

$\frac{\mathrm{d} X}{\mathrm{~d} t}=\mu_{\max } X\left(1-\frac{X}{X_{\mathrm{m}}}\right)$.

On integration, the above equation gives the logistic (L)type model equation that relates hyperbolic growth of cell:

$X(t)=\frac{X_{0} e^{\mu_{\max } t}}{1-\frac{X_{0}}{X_{\mathrm{m}}}\left(1-e^{\mu_{\max } t}\right)}$,

where $X$ is biomass concentration, $\mathrm{g} / \mathrm{l}, \mu_{\max }$ is the maximum specific cell growth rate, day ${ }^{-1}$, and $X_{\mathrm{m}}$ is the maximum biomass concentration, $\mathrm{g} / \mathrm{l}$.

Bioactive metabolite production can be obtained from growth limiting substrate (optimized media constituents) and the substrate utilization kinetics can be taken from Modified Leudeking-Piret (MLP) equation:

$-\frac{\mathrm{d} S}{\mathrm{~d} t}=r_{\mathrm{S}}=\gamma\left(\frac{\mathrm{d} X}{\mathrm{~d} t}\right)+\eta X$.

On integration, the above equation results logistic incorporated modified Leudeking-Piret (LIMLP) equation:

$$
\begin{aligned}
S(t)= & S_{0}-\gamma\left[\frac{X_{0} e^{\mu_{\max } t}}{1-\left(\frac{X_{0}}{X_{\mathrm{m}}}\right)\left(1-e^{\mu_{\max } t}\right)}-X_{0}\right] \\
& +\frac{\eta X_{\mathrm{m}}}{\mu_{\max }} \ln \left[1-\left(\frac{X_{0}}{X_{\mathrm{m}}}\right)\left(1-e^{\mu_{\max } t}\right)\right] .
\end{aligned}
$$

Constant of non-growth-associated substrate consumption, $\eta$, in above equation can be calculated from stationary phase data(where $\left.\frac{-\mathrm{d} S}{\mathrm{~d} t}=0\right)$ :

$\eta=\frac{-\left(\frac{\mathrm{d} S}{\mathrm{~d} t}\right)_{\text {stationaryphase }}}{X_{\max }}$.

Significant bioactive metabolite (product) formation occurs in late-logarithmic phase of cell growth and bioactive metabolite formation kinetics follows Leudeking-Piret equation (Luedeking and Piret 2000), as: 
$\frac{\mathrm{d} P}{\mathrm{~d} t}=\alpha \frac{\mathrm{d} X}{\mathrm{~d} t}+\beta X$.

Logistic incorporated Leudeking-Piret (LILP) equation derived from integration of the above equation results:

$$
\begin{aligned}
P(t)= & P_{0}+\alpha\left[\frac{X_{0} e^{\mu_{\max } t}}{1-\left(\frac{X_{0}}{X_{\mathrm{m}}}\right)\left(1-e^{\mu_{\max } t}\right)}-X_{0}\right] \\
& +\frac{\beta X_{\mathrm{m}}}{\mu} \ln \left[1-\left(\frac{X_{0}}{X_{\mathrm{m}}}\right)\left(1-e^{\mu_{\max } t}\right)\right] .
\end{aligned}
$$

Non-growth-associated product formation constant, $\beta$, can be determined from stationary phase data (where $\left.\frac{\mathrm{d} X}{\mathrm{~d} t}=0\right)$ :

$\beta=\frac{\left(\frac{\mathrm{d} P}{\mathrm{~d} t}\right)_{\text {stationaryphase }}}{X_{\max }}$.

Experimental data obtained from batch shake-flask fermentations was used to simulate using Eqs. (4), (6), and (9).

\section{Results}

\section{RSM modeling}

Influence of different physico-chemical parameters on bioactive metabolite production and their effect on the response (measured as inhibition zones) were investigated and optimized as per the model designed by CCD of RSM.
The effect of independent variable optimization on the responses was identified by complete five factors and threelevel factorial experiment designs with eight replications of central point and ten axial points and thirty-two factorial points for bioactive metabolite production by the strain. The maximum production of the bioactive compound and its effect on the responses (inhibition of growth of the pathogenic microorganisms by the bioactive compound produced by $N$. litoralis strain VSM 8 is represented in $\mathrm{mm}$ ) was experimentally found to be $21 \mathrm{~mm}$ (Staphylococcus aureus), $20 \mathrm{~mm}$ (Bacillus subtilis), $22.9 \mathrm{~mm}$ (X. campestris), $19.9 \mathrm{~mm}$ (Pseudomonas aeruginosa), and $17.9 \mathrm{~mm}$ (Candida albicans) that was obtained from cultural conditions of the strain grown in a medium containing $2 \%$ sucrose, $1 \%$ soya peptone with $\mathrm{pH} 8$ incubated at $25{ }^{\circ} \mathrm{C}$ for 11 days.

To determine whether the model would give misleading or approximate results, the experimental data are subjected to model adequacy. Linear, interactive, quadratic, and cubic models were fitted to the experimental data to determine the actual relationship between the response and the variables selected for the study. The suggested sequential model sum of squares and lack of fit tests (showing degrees of freedom; mean square, $F$ value, $p$ value), model summary statistics (showing standard deviation, $R^{2}$, adjusted $R^{2}$ and predicted $R^{2}$ ) for the best outcome quadratic model (Bipasha et al. 2015), for all the five responses, are given in Table 2.

As per the sequential model sum of squares of all the five responses, the quadratic model was significant with

\begin{tabular}{|c|c|c|c|c|c|}
\hline Model parameter & S. aureus & B. subtilis & X. campestris & P. aeruginosa & C. albicans \\
\hline \multicolumn{6}{|c|}{ Sequential model sum of squares—quadratic vs $2 \mathrm{FI}$ (suggested) } \\
\hline Sum of squares & 85.83 & 59.22 & 237.14 & 94.56 & 26.82 \\
\hline$d f$ & 5 & 5 & 5 & 5 & 5 \\
\hline Mean square & 11.17 & 11.84 & 47.43 & 18.91 & 5.36 \\
\hline$F$ value & 2157.78 & 240.13 & 982.19 & 317.08 & 590.81 \\
\hline$p$ value $($ Prob $>F)$ & $<0.0001$ & $<0.0001$ & $<0.0001$ & $<0.0001$ & $<0.0001$ \\
\hline \multicolumn{6}{|c|}{ Lack of fit tests-quadratic (suggested) } \\
\hline Sum of squares & 0.15 & 0.71 & 1.40 & 1.73 & 0.26 \\
\hline$d f$ & 20 & 20 & 20 & 20 & 25 \\
\hline Mean square & $7.535 \mathrm{E}-003$ & 0.036 & 0.0070 & 0.086 & 1.08 \\
\hline$F$ value & 0.85 & 0.44 & - & - & - \\
\hline$p$ value $($ Prob $>F)$ & 0.6410 & 0.9373 & - & - & - \\
\hline \multicolumn{6}{|c|}{ Model summary statistics—quadratic (suggested) } \\
\hline Std. Dev. & 0.089 & 0.22 & 0.22 & 0.24 & 0.095 \\
\hline$R^{2}$ & 0.9975 & 0.9797 & 0.9944 & 0.9824 & 0.9925 \\
\hline Adjusted $R^{2}$ & 0.9957 & 0.9657 & 0.9906 & 0.9702 & 0.9873 \\
\hline Predicted $R^{2}$ & 0.9931 & 0.9503 & 0.9811 & 0.9551 & 0.9781 \\
\hline
\end{tabular}

Table 2 Sequential model fitting for all the responses (in terms of inhibition zone produced by bioactive metabolite)

$d f$ degrees of freedom 


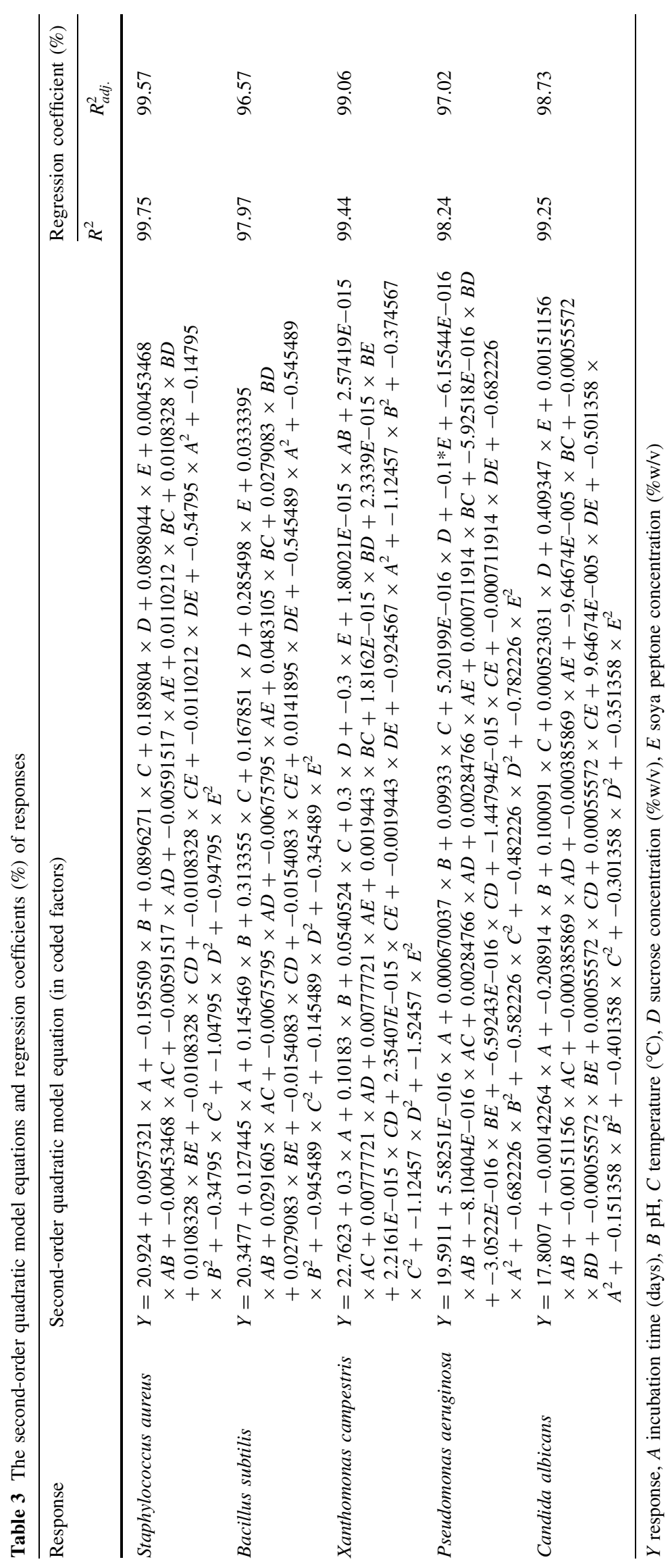


Table 4 ANOVA for Staphylococcus aureus response surface quadratic model

\begin{tabular}{|c|c|c|c|c|c|c|}
\hline Source & Sum of squares & $d f^{\mathrm{a}}$ & Mean square & $F$ value & $P$ value Prob $>F$ & \\
\hline Model & 90.57 & 20 & 4.53 & 569.25 & $<0.0001$ & Significant \\
\hline$A$-time of incubation & 0.28 & 1 & 0.28 & 35.02 & $<0.0001$ & \\
\hline$B-\mathrm{pH}$ & 1.26 & 1 & 1.26 & 157.88 & $<0.0001$ & \\
\hline$C$-temperature & 0.26 & 1 & 0.26 & 33.18 & $<0.0001$ & \\
\hline$D$-Sucrose & 1.16 & 1 & 1.16 & 145.35 & $<0.0001$ & \\
\hline E-Soya Peptone & 0.26 & 1 & 0.26 & 32.54 & $<0.0001$ & \\
\hline$A B$ & $5.844 \mathrm{E}-004$ & 1 & $5.844 \mathrm{E}-004$ & 0.073 & 0.7883 & \\
\hline$A C$ & $5.844 \mathrm{E}-004$ & 1 & $5.844 \mathrm{E}-004$ & 0.073 & 0.7883 & \\
\hline$A D$ & $1.027 \mathrm{E}-003$ & 1 & $1.027 \mathrm{E}-003$ & 0.13 & 0.7219 & \\
\hline$A E$ & $1.027 \mathrm{E}-003$ & 1 & $1.027 \mathrm{E}-003$ & 0.13 & 0.7219 & \\
\hline$B C$ & $3.749 \mathrm{E}-003$ & 1 & $3.749 \mathrm{E}-003$ & 0.47 & 0.4979 & \\
\hline$B D$ & $3.533 \mathrm{E}-003$ & 1 & $3.533 \mathrm{E}-003$ & 0.44 & 0.5104 & \\
\hline$B E$ & $3.533 \mathrm{E}-003$ & 1 & $3.533 \mathrm{E}-003$ & 0.44 & 0.5104 & \\
\hline$C D$ & $3.533 \mathrm{E}-003$ & 1 & $3.533 \mathrm{E}-003$ & 0.44 & 0.5104 & \\
\hline$C E$ & $3.533 \mathrm{E}-003$ & 1 & $3.533 \mathrm{E}-003$ & 0.44 & 0.5104 & \\
\hline$D E$ & $3.749 \mathrm{E}-003$ & 1 & $3.749 \mathrm{E}-003$ & 0.47 & 0.4979 & \\
\hline$A^{2}$ & 0.74 & 1 & 0.74 & 93.34 & $<0.0001$ & \\
\hline$B^{2}$ & 0.054 & 1 & 0.054 & 6.80 & 0.0142 & \\
\hline$C^{2}$ & 0.30 & 1 & 0.30 & 37.64 & $<0.0001$ & \\
\hline$D^{2}$ & 2.72 & 1 & 2.72 & 341.39 & $<0.0001$ & \\
\hline$E^{2}$ & 2.22 & 1 & 2.22 & 279.34 & $<0.0001$ & \\
\hline
\end{tabular}

${ }^{a}$ Degrees of freedom

Table 5 ANOVA for Bacillus subtilis response surface quadratic model

\begin{tabular}{|c|c|c|c|c|c|c|}
\hline Source & Sum of squares & $d f^{a}$ & Mean square & $F$ value & $P$ value $\operatorname{Prob}>F$ & \\
\hline Model & 68.97 & 20 & 3.45 & 69.91 & $<0.0001$ & Significant \\
\hline$A$-time of incubation & 0.49 & 1 & 0.49 & 10.01 & 0.0036 & \\
\hline$B-\mathrm{pH}$ & 0.70 & 1 & 0.70 & 14.10 & 0.0008 & \\
\hline$C$-temperature & 3.23 & 1 & 3.23 & 65.41 & $<0.0001$ & \\
\hline$D$-Sucrose & 0.90 & 1 & 0.90 & 18.33 & 0.0002 & \\
\hline$E$-Soya peptone & 2.62 & 1 & 2.62 & 53.04 & $<0.0001$ & \\
\hline$A B$ & 0.0321 & 1 & 0.032 & 0.64 & 0.4301 & \\
\hline$A C$ & 0.024 & 1 & 0.024 & 0.49 & 0.4895 & \\
\hline$A D$ & $1.341 \mathrm{E}-003$ & 1 & $1.341 \mathrm{E}-003$ & 0.027 & 0.8702 & \\
\hline$A E$ & $1.341 \mathrm{E}-003$ & 1 & $1.341 \mathrm{E}-003$ & 0.027 & 0.8702 & \\
\hline$B C$ & 0.072 & 1 & 0.072 & 1.46 & 0.2366 & \\
\hline$B D$ & 0.023 & 1 & 0.023 & 0.48 & 0.4960 & \\
\hline$B E$ & 0.023 & 1 & 0.023 & 0.48 & 0.4960 & \\
\hline$C D$ & $7.147 \mathrm{E}-003$ & 1 & $7.417 \mathrm{E}-003$ & 0.14 & 0.7062 & \\
\hline$C E$ & $7.147 \mathrm{E}-003$ & 1 & $7.147 \mathrm{E}-003$ & 0.14 & 0.7062 & \\
\hline$D E$ & $6.214 \mathrm{E}-003$ & 1 & $6.214 \mathrm{E}-003$ & 0.13 & 0.7252 & \\
\hline$A^{2}$ & 0.74 & 1 & 0.74 & 14.92 & 0.0006 & \\
\hline$B$ & 0.74 & 1 & 0.74 & 14.92 & 0.0006 & \\
\hline$C^{2}$ & 2.21 & 1 & 2.21 & 44.82 & $<0.0001$ & \\
\hline$D^{2}$ & 0.052 & 1 & 0.052 & 1.06 & 0.3114 & \\
\hline$E^{2}$ & 0.30 & 1 & 0.30 & 5.98 & 0.0207 & \\
\hline
\end{tabular}

${ }^{a}$ Degrees of freedom 
Table 6 ANOVA for Xanthomonas campestris response surface quadratic model

\begin{tabular}{|c|c|c|c|c|c|c|}
\hline Source & Sum of squares & $d f^{\mathrm{a}}$ & Mean square & $F$ value & $P$ value Prob $>F$ & \\
\hline Model & 249.64 & 20 & 12.48 & 258.49 & $<0.0001$ & Significant \\
\hline$A$-time of incubation & 2.74 & 1 & 22.74 & 56.65 & $<0.0001$ & \\
\hline$B-\mathrm{pH}$ & 0.34 & 1 & 0.34 & 7.06 & 0.0127 & \\
\hline$C$-temperature & 0.096 & 1 & 0.096 & 1.99 & 0.1692 & \\
\hline$D$-Sucrose & 2.89 & 1 & 2.89 & 59.82 & $<0.0001$ & \\
\hline$E$-Soya peptone & 2.89 & 1 & 2.89 & 59.82 & $<0.0001$ & \\
\hline$A B$ & 0.000 & 1 & 0.000 & 0.000 & 1.0000 & \\
\hline$A C$ & 0.000 & 1 & 0.000 & 0.000 & 1.0000 & \\
\hline$A D$ & $1.776 \mathrm{E}-003$ & 1 & $1.776 \mathrm{E}-003$ & 0.037 & 0.8493 & \\
\hline$A E$ & $1.776 \mathrm{E}-003$ & 1 & $1.776 \mathrm{E}-003$ & 0.37 & 0.8493 & \\
\hline$B C$ & $1.167 \mathrm{E}-003$ & 1 & $1.167 \mathrm{E}-003$ & $2.416 \mathrm{E}-003$ & 0.9611 & \\
\hline$B D$ & 0.000 & 1 & 0.000 & 0.000 & 1.0000 & \\
\hline$B E$ & 0.000 & 1 & 0.000 & 0.000 & 1.0000 & \\
\hline$C D$ & 0.000 & 1 & 0.000 & 0.000 & 1.0000 & \\
\hline$C E$ & 0.000 & 1 & 0.000 & 0.000 & 1.0000 & \\
\hline$D E$ & $1.167 \mathrm{E}-003$ & 1 & $1.167 \mathrm{E}-003$ & $2.416 \mathrm{E}-003$ & 0.9611 & \\
\hline$A^{2}$ & 2.11 & 1 & 2.11 & 43.78 & $<0.0001$ & \\
\hline$B^{2}$ & 3.13 & 1 & 3.13 & 64.77 & $<0.0001$ & \\
\hline$C^{2}$ & 0.35 & 1 & 0.35 & 7.19 & 0.0120 & \\
\hline$D^{2}$ & 3.13 & 1 & 3.13 & 64.77 & $<0.0001$ & \\
\hline$E^{2}$ & 5.75 & 1 & 5.75 & 119.03 & $<0.0001$ & \\
\hline
\end{tabular}

${ }^{a}$ Degrees of freedom

Table 7 ANOVA for Pseudomonas aeruginosa response surface quadratic model

\begin{tabular}{|c|c|c|c|c|c|c|}
\hline Source & Sum of squares & $d f^{a}$ & Mean square & $F$ value & $P$ value Prob $>F$ & \\
\hline Model & 96.43 & 20 & 4.82 & 80.83 & $<0.0001$ & Significant \\
\hline$A$-time of incubation & 0.000 & 1 & 0.000 & & 1.0000 & \\
\hline$B-\mathrm{pH}$ & $1.475 \mathrm{E}-005$ & 1 & $1.475 \mathrm{E}-005$ & $2.473 \mathrm{E}-004$ & 0.9876 & \\
\hline$C$-temperature & 0.32 & 1 & 0.32 & 5.44 & 0.0269 & \\
\hline$D$-Sucrose & 0.000 & 1 & 0.000 & 0.000 & 1.0000 & \\
\hline$E$-Soya peptone & 0.32 & 1 & 0.32 & 5.38 & 0.0276 & \\
\hline$A B$ & 0.000 & 1 & 0.000 & 0.000 & 1.0000 & \\
\hline$A C$ & 0.000 & 1 & 0.000 & 0.000 & 1.0000 & \\
\hline$A D$ & $2.381 \mathrm{E}-004$ & 1 & $2.381 \mathrm{E}-004$ & $3.992 \mathrm{E}-003$ & 0.9501 & \\
\hline$A E$ & $2.381 \mathrm{E}-004$ & 1 & $2.381 \mathrm{E}-004$ & $3.992 \mathrm{E}-003$ & 0.9501 & \\
\hline$B C$ & $1.564 \mathrm{E}-005$ & 1 & $1.564 \mathrm{E}-005$ & $2.622 \mathrm{E}-004$ & 0.9872 & \\
\hline$B D$ & 0.000 & 1 & 0.000 & 0.000 & 1.0000 & \\
\hline$B E$ & 0.000 & 1 & 0.000 & 0.000 & 1.0000 & \\
\hline$C D$ & 0.000 & 1 & 0.000 & 0.000 & 1.0000 & \\
\hline$C E$ & 0.000 & 1 & 0.000 & 0.000 & 1.0000 & \\
\hline$D E$ & $1.564 \mathrm{E}-005$ & 1 & $1.564 \mathrm{E}-005$ & $2.622 \mathrm{E}-004$ & 0.9872 & \\
\hline$A^{2}$ & 1.15 & 1 & 1.15 & 19.30 & 0.0001 & \\
\hline$B^{2}$ & 1.15 & 1 & 1.15 & 19.30 & 0.0001 & \\
\hline$C^{2}$ & 0.84 & 1 & 0.84 & 14.05 & 0.0008 & \\
\hline$D^{2}$ & 0.58 & 1 & 0.58 & 9.64 & 0.0042 & \\
\hline$E^{2}$ & 1.15 & 1 & 1.15 & 25.37 & $<0.0001$ & \\
\hline
\end{tabular}

${ }^{a}$ Degrees of freedom 
Table 8 ANOVA for Candida albicans response surface quadratic model

\begin{tabular}{|c|c|c|c|c|c|c|}
\hline Source & Sum of squares & $d f^{\mathrm{a}}$ & Mean square & $F$ value & $P$ value Prob $>F$ & \\
\hline Model & 34.68 & 20 & 1.73 & 190.97 & $<0.0001$ & Significant \\
\hline$A$-time of incubation & $6.152 \mathrm{E}-005$ & 1 & $6.152 \mathrm{E}-005$ & $6.776 \mathrm{E}-003$ & 0.9350 & \\
\hline$B-\mathrm{pH}$ & 1.43 & 1 & 1.43 & 157.96 & $<0.0001$ & \\
\hline$C$-temperature & 0.33 & 1 & 0.33 & 36.26 & $<0.0001$ & \\
\hline$D$-Sucrose & $8.781 \mathrm{E}-006$ & 1 & $8.781 \mathrm{E}-006$ & $9.671 \mathrm{E}-004$ & 0.9754 & \\
\hline$E$-Soya peptone & 5.38 & 1 & 5.38 & 592.38 & $<0.0001$ & \\
\hline$A B$ & $6.493 \mathrm{E}-005$ & 1 & $6.493 \mathrm{E}-005$ & $7.152 \mathrm{E}-003$ & 0.9332 & \\
\hline$A C$ & $6.493 \mathrm{E}-005$ & 1 & $6.493 \mathrm{E}-005$ & $7.152 \mathrm{E}-003$ & 0.9332 & \\
\hline$A D$ & $4.372 \mathrm{E}-005$ & 1 & $4.372 \mathrm{E}-005$ & $4815 \mathrm{E}-004$ & 0.9826 & \\
\hline$A E$ & $4.372 \mathrm{E}-005$ & 1 & $4.372 \mathrm{E}-005$ & $4815 \mathrm{E}-004$ & 0.9826 & \\
\hline$B C$ & $2.872 \mathrm{E}-007$ & 1 & $2.872 \mathrm{E}-007$ & $3.163 \mathrm{E}-005$ & 0.9956 & \\
\hline$B D$ & $9.297 \mathrm{E}-006$ & 1 & $9.297 \mathrm{E}-006$ & $1.024 \mathrm{E}-003$ & 0.9747 & \\
\hline$B E$ & $9.297 \mathrm{E}-006$ & 1 & $9.297 \mathrm{E}-006$ & $1.024 \mathrm{E}-003$ & 0.9747 & \\
\hline$C D$ & $9.297 \mathrm{E}-006$ & 1 & $9.297 \mathrm{E}-006$ & $1.024 \mathrm{E}-003$ & 0.9747 & \\
\hline$C E$ & $9.297 \mathrm{E}-006$ & 1 & $9.297 \mathrm{E}-006$ & $1.024 \mathrm{E}-003$ & 0.9747 & \\
\hline$D E$ & $2.872 \mathrm{E}-007$ & 1 & $2.872 \mathrm{E}-007$ & $3.163 \mathrm{E}-005$ & 0.9956 & \\
\hline$A^{2}$ & 0.62 & 1 & 0.62 & 68.47 & $<0.0001$ & \\
\hline$B^{2}$ & 0.057 & 1 & 0.057 & 6.24 & 0.0184 & \\
\hline$C^{2}$ & 0.40 & 1 & 0.40 & 43.88 & $<0.0001$ & \\
\hline$D^{2}$ & 0.22 & 1 & 0.22 & 24.74 & $<0.0001$ & \\
\hline$E^{2}$ & 0.31 & 1 & 0.31 & 33.63 & $<0.0001$ & \\
\hline
\end{tabular}

${ }^{a}$ Degrees of freedom

( $p$ value $<0.0001$ ) for responses Staphylococcus aureus, Bacillus subtilis, Xanthomonas campestris, Pseudomonas aeruginosa, and Candida albicans (inhibition of the growth was represented in $\mathrm{mm}$ ). The lack of fit test values of quadratic models identified earlier as the accepted model for all the responses was found to be insignificant lack of fit which indicate that the model is highly significant. The second-order quadratic model equations and regression coefficients (\%) of all responses (Urailuck et al. 2015) were given in Table 3 .

The model was found to be statistically significant with confidence level of $99.9 \%$ since the Prob $>F$ value of the model for all the five responses was found to be $<0.00001$ and $F$ values of the model for all the five responses was found to be $<0.00001$ and $F$ values of the model for five responses 569.25, 69.91, 258.49, 80.83, and 190.97 (in terms of inhibition of growth of the pathogenic microorganisms, viz., Staphylococcus aureus, Bacillus subtilis, Xanthomonas campestris, Pseudomonas aeruginosa, Candida albicans, respectively, by the bioactive compound produced by VSM 8 is represented in $\mathrm{mm}$ ) implies that the model is significant. Model terms are said to be significant if the values of $p$ (Prob $<F$ ) is less than 0.0500 . ANOVA reveals that most of the significant factors affecting the growth inhibition of pathogenic microorganisms (five responses) by the bioactive compounds produced by VSM 8 for the variables: incubation time (days), $\mathrm{pH}$, temperature, concentration of sucrose, and concentration of soya peptone. Tables $4,5,6,7$ and 8 show the ANOVA results obtained from statistical optimization.

Regression analysis indicated that the coefficient of determination $\left(R^{2}\right)$ values of the five responses and high value of $R^{2}$ indicate that the full quadratic model equation was capable of representing that the system under a given 
Table 9 Central composite factor experimental design along with experimental and predicted values

\begin{tabular}{|c|c|c|c|c|c|c|c|c|c|c|c|c|c|c|c|}
\hline \multirow[t]{2}{*}{ Run } & \multirow[t]{2}{*}{$A$-time (days) } & \multirow[t]{2}{*}{$B-\mathrm{pH}$} & \multirow[t]{2}{*}{$C$-temperature $\left({ }^{\circ} \mathrm{C}\right)$} & \multirow{2}{*}{$\begin{array}{l}D \text {-[Sucrose }] \\
(\% \mathrm{w} / \mathrm{v})\end{array}$} & \multirow{2}{*}{$\begin{array}{l}E \text {-[Soya peptone] } \\
(\% \mathrm{w} / \mathrm{v})\end{array}$} & \multicolumn{2}{|c|}{ S. aureus } & \multicolumn{2}{|c|}{ B. subtilis } & \multicolumn{2}{|c|}{$X$. campestris } & \multicolumn{2}{|c|}{ P. aeruginosa } & \multicolumn{2}{|c|}{ C. albicans } \\
\hline & & & & & & Actual & RSM & Actual & RSM & Actual & RSM & Actual & RSM & Actual & RSM \\
\hline 1 & 11.00 & 7.00 & 25.00 & 2.00 & 1.00 & 17.60 & 17.60 & 16.80 & 16.92 & 17.20 & 17.20 & 16.40 & 16.40 & 15.80 & 15.80 \\
\hline 2 & 10.00 & 9.00 & 30.00 & 1.00 & 0.50 & 17.80 & 17.82 & 17.00 & 17.07 & 17.80 & 17.80 & 16.40 & 16.40 & 15.80 & 15.80 \\
\hline 3 & 11.00 & 8.00 & 25.00 & 2.00 & 1.50 & 17.20 & 17.15 & 17.00 & 16.93 & 17.40 & 17.40 & 16.40 & 16.40 & 15.40 & 15.40 \\
\hline 4 & 11.00 & 8.00 & 25.00 & 2.00 & 1.00 & 17.40 & 17.38 & 17.20 & 17.22 & 18.00 & 18.00 & 16.40 & 16.40 & 15.40 & 15.40 \\
\hline 5 & 10.00 & 9.00 & 20.00 & 1.00 & 1.50 & 17.80 & 17.81 & 17.40 & 17.45 & 17.40 & 17.40 & 16.60 & 16.60 & 16.00 & 16.00 \\
\hline 6 & 10.00 & 9.00 & 20.00 & 1.00 & 0.50 & 17.80 & 17.81 & 17.40 & 17.45 & 17.40 & 17.40 & 16.60 & 16.60 & 16.00 & 16.00 \\
\hline 7 & 12.00 & 9.00 & 20.00 & 1.00 & 1.50 & 17.40 & 17.40 & 17.60 & 17.33 & 17.60 & 17.60 & 16.60 & 16.60 & 15.60 & 15.60 \\
\hline 8 & 11.00 & 8.00 & 25.00 & 1.00 & 1.00 & 17.60 & 17.62 & 18.20 & 18.07 & 18.20 & 18.20 & 16.60 & 16.60 & 15.60 & 15.60 \\
\hline 9 & 12.00 & 7.00 & 20.00 & 1.00 & 1.50 & 18.00 & 18.02 & 17.20 & 17.21 & 17.80 & 17.80 & 16.40 & 16.40 & 15.80 & 15.80 \\
\hline 10 & 11.00 & 9.00 & 25.00 & 2.00 & 1.00 & 18.00 & 18.21 & 17.40 & 17.34 & 18.40 & 18.40 & 16.40 & 16.40 & 15.80 & 15.80 \\
\hline 11 & 12.00 & 9.00 & 30.00 & 1.00 & 0.50 & 17.60 & 17.61 & 17.40 & 17.34 & 18.00 & 18.00 & 16.40 & 16.40 & 15.40 & 15.40 \\
\hline 12 & 11.00 & 8.00 & 25.00 & 2.00 & 1.00 & 17.80 & 17.81 & 17.60 & 17.60 & 18.60 & 18.60 & 16.40 & 16.40 & 15.40 & 15.40 \\
\hline 13 & 10.00 & 7.00 & 20.00 & 3.00 & 0.50 & 18.20 & 18.18 & 17.80 & 17.68 & 18.00 & 18.00 & 16.60 & 16.60 & 16.00 & 16.00 \\
\hline 14 & 10.00 & 7.00 & 20.00 & 1.00 & 0.50 & 18.40 & 18.35 & 18.00 & 17.93 & 18.60 & 18.60 & 16.60 & 16.60 & 16.00 & 16.00 \\
\hline 15 & 12.00 & 9.00 & 30.00 & 3.00 & 0.50 & 17.80 & 17.82 & 18.00 & 18.01 & 18.20 & 18.20 & 16.60 & 16.60 & 15.60 & 15.60 \\
\hline 16 & 12.00 & 7.00 & 30.00 & 1.00 & 1.50 & 18.00 & 18.01 & 18.20 & 18.39 & 18.80 & 18.80 & 16.60 & 16.60 & 15.60 & 15.60 \\
\hline 17 & 12.00 & 8.00 & 25.00 & 2.00 & 1.00 & 17.80 & 17.82 & 17.40 & 17.45 & 16.60 & 16.60 & 16.20 & 16.20 & 16.60 & 16.60 \\
\hline 18 & 10.00 & 7.00 & 20.00 & 3.00 & 1.50 & 18.00 & 18.01 & 17.60 & 17.58 & 17.20 & 17.20 & 16.20 & 16.20 & 16.60 & 16.60 \\
\hline 19 & 11.00 & 8.00 & 25.00 & 2.00 & 1.00 & 17.40 & 17.41 & 17.60 & 17.58 & 16.80 & 16.80 & 16.20 & 16.20 & 16.20 & 16.20 \\
\hline 20 & 11.00 & 8.00 & 20.00 & 2.00 & 1.00 & 17.60 & 17.61 & 17.80 & 17.84 & 17.40 & 17.40 & 16.20 & 16.20 & 16.20 & 16.20 \\
\hline 21 & 10.00 & 9.00 & 30.00 & 1.00 & 1.50 & 18.00 & 17.98 & 18.00 & 17.92 & 16.80 & 16.80 & 16.40 & 16.40 & 16.80 & 16.80 \\
\hline 22 & 10.00 & 8.00 & 25.00 & 2.00 & 1.00 & 18.20 & 18.15 & 18.20 & 18.17 & 17.40 & 17.40 & 16.40 & 16.40 & 16.80 & 16.80 \\
\hline 23 & 10.00 & 9.00 & 20.00 & 3.00 & 1.50 & 17.60 & 17.62 & 18.20 & 18.24 & 17.00 & 17.00 & 16.40 & 16.40 & 16.40 & 16.40 \\
\hline 24 & 12.00 & 7.00 & 20.00 & 3.00 & 1.50 & 17.80 & 17.81 & 18.40 & 18.62 & 17.60 & 17.60 & 16.40 & 16.40 & 16.40 & 16.40 \\
\hline 25 & 12.00 & 9.00 & 20.00 & 3.00 & 0.50 & 18.20 & 18.18 & 17.80 & 17.80 & 17.20 & 17.20 & 16.20 & 16.20 & 16.60 & 16.60 \\
\hline 26 & 10.00 & 9.00 & 30.00 & 3.00 & 0.50 & 18.40 & 18.15 & 18.00 & 17.90 & 17.80 & 17.80 & 16.20 & 16.20 & 16.60 & 16.60 \\
\hline 27 & 12.00 & 9.00 & 20.00 & 3.00 & 1.50 & 17.80 & 17.82 & 18.00 & 18.04 & 17.40 & 17.40 & 16.20 & 16.20 & 16.20 & 16.20 \\
\hline 28 & 10.00 & 7.00 & 30.00 & 1.00 & 1.50 & 18.00 & 18.00 & 18.20 & 18.28 & 18.00 & 18.00 & 16.20 & 16.20 & 16.20 & 16.20 \\
\hline 29 & 12.00 & 7.00 & 30.00 & 3.00 & 0.50 & 18.60 & 18.46 & 18.00 & 18.43 & 18.00 & 18.00 & 16.40 & 16.40 & 16.80 & 1.80 \\
\hline 30 & 12.00 & 7.00 & 30.00 & 3.00 & 1.50 & 18.20 & 18.46 & 18.60 & 18.43 & 18.00 & 18.00 & 16.40 & 16.40 & 16.80 & 16.80 \\
\hline 31 & 11.00 & 8.00 & 25.00 & 2.00 & 1.00 & 18.00 & 17.98 & 18.60 & 18.64 & 17.60 & 17.60 & 16.40 & 16.40 & 16.40 & 16.40 \\
\hline 32 & 12.00 & 7.00 & 30.00 & 3.00 & 1.50 & 18.20 & 18.15 & 19.40 & 19.00 & 18.20 & 18.20 & 16.40 & 16.40 & 16.40 & 16.40 \\
\hline 33 & 10.00 & 7.00 & 30.00 & 1.00 & 0.50 & 20.20 & 20.20 & 19.80 & 19.67 & 21.40 & 21.40 & 18.60 & 18.60 & 17.20 & 17.20 \\
\hline 34 & 10.00 & 9.00 & 30.00 & 3.00 & 1.50 & 20.40 & 20.39 & 20.00 & 19.93 & 22.00 & 22.00 & 18.60 & 18.60 & 17.20 & 17.20 \\
\hline 35 & 11.00 & 8.00 & 25.00 & 2.00 & 1.00 & 20.80 & 20.79 & 19.80 & 19.66 & 21.40 & 21.40 & 18.60 & 18.60 & 17.60 & 17.60 \\
\hline 36 & 12.00 & 9.00 & 30.00 & 3.00 & 1.50 & 20.40 & 20.41 & 20.00 & 19.95 & 21.60 & 21.60 & 18.60 & 18.60 & 17.20 & 17.20 \\
\hline 37 & 11.00 & 8.00 & 25.00 & 2.00 & 0.50 & 20.40 & 20.41 & 19.20 & 19.09 & 21.40 & 21.40 & 18.60 & 18.60 & 17.20 & 17.20 \\
\hline 38 & 12.00 & 9.00 & 20.00 & 1.00 & 0.50 & 20.60 & 20.59 & 19.80 & 19.72 & 21.60 & 21.60 & 18.80 & 18.80 & 17.40 & 17.40 \\
\hline 39 & 11.00 & 8.00 & 30.00 & 2.00 & 1.00 & 19.60 & 19.61 & 20.40 & 20.03 & 21.20 & 21.20 & 18.80 & 18.80 & 17.40 & 17.40 \\
\hline 40 & 12.00 & 9.00 & 30.00 & 1.00 & 1.50 & 20.00 & 19.99 & 20.20 & 20.37 & 21.80 & 21.80 & 18.80 & 18.80 & 17.40 & 17.40 \\
\hline 41 & 11.00 & 8.00 & 25.00 & 3.00 & 1.00 & 19.80 & 19.81 & 19.80 & 19.72 & 21.40 & 21.40 & 18.60 & 18.60 & 16.80 & 16.80 \\
\hline 42 & 11.00 & 8.00 & 25.00 & 2.00 & 1.00 & 20.00 & 19.99 & 20.40 & 20.29 & 20.80 & 20.80 & 18.40 & 18.40 & 17.60 & 17.60 \\
\hline 43 & 10.00 & 9.00 & 20.00 & 3.00 & 0.50 & 20.80 & 20.80 & 20.40 & 20.35 & 22.40 & 22.40 & 19.20 & 19.20 & 17.60 & 17.60 \\
\hline 44 & 10.00 & 7.00 & 30.00 & 1.00 & 0.50 & 20.80 & 20.80 & 19.80 & 20.35 & 22.40 & 22.40 & 19.20 & 19.20 & 17.60 & 17.60 \\
\hline 45 & 12.00 & 7.00 & 20.00 & 1.00 & 0.50 & 20.80 & 20.80 & 20.40 & 20.35 & 22.40 & 22.40 & 19.20 & 19.20 & 17.60 & 17.60 \\
\hline 46 & 10.00 & 7.00 & 30.00 & 3.00 & 0.50 & 20.80 & 20.80 & 20.40 & 20.35 & 22.40 & 22.40 & 19.20 & 19.20 & 17.60 & 17.60 \\
\hline 47 & 12.00 & 7.00 & 20.00 & 3.00 & 0.50 & 20.80 & 20.80 & 19.80 & 20.35 & 22.40 & 22.40 & 19.20 & 19.20 & 17.60 & 17.60 \\
\hline 48 & 11.00 & 8.00 & 25.00 & 2.00 & 1.00 & 20.80 & 20.80 & 20.40 & 20.35 & 22.40 & 22.40 & 19.20 & 19.20 & 17.60 & 17.60 \\
\hline 49 & 11.00 & 8.00 & 25.00 & 2.00 & 1.00 & 20.80 & 20.80 & 20.40 & 20.35 & 22.40 & 22.40 & 19.20 & 19.20 & 17.60 & 17.60 \\
\hline 50 & 10.00 & 7.00 & 20.00 & 1.00 & 1.50 & 20.80 & 20.80 & 20.40 & 20.35 & 22.40 & 22.40 & 19.20 & 19.20 & 17.60 & 17.60 \\
\hline
\end{tabular}


experimental domain is significant and adjusted coefficient of determination $\left(R_{a d j}^{2}\right.$. ) values also indicated good agreement between experimental and the predicted values. Degree of precision is indicated by the coefficient variation (CV) with which the experiment is compared. Experimental reliability is poor if the $\mathrm{CV}$ value is high. The $\mathrm{CV}$ (\%) values of the five responses in this study were found to be $0.47,1.19,1.14,1.4$, and 0.57 which denote that the performed experiment is reliable. The present model was used to evaluate the direct interaction and quadratic effects to optimize the physico-chemical parameters for the production and bioactive metabolites that inhibit the pathogenic microorganisms (responses). Central composite factor experimental design along with experimental and predicted values is shown in Table 9.

\section{Quadratic and interactive effects of bioactive metabolite production and its effect on the responses}

The effect of individual parameters, such as time of incubation (in days), $\mathrm{pH}$, temperature, concentration of sucrose, and concentration of soya peptone on the responses, are insignificant, but exhibited significant interactions with other parameters. The significant interactive effects of the variables (incubation time- $\mathrm{pH}$, incubation time-temperature, incubation time-concentration of sucrose, incubation time-concentration of soya peptone, $\mathrm{pH}$-temperature, $\mathrm{pH}-$ concentration of sucrose, $\mathrm{pH}$-concentration of soya peptone, temperature-concentration of sucrose, temperatureconcentration of soya peptone, and concentration of sucrose-concentration of soya peptone) are represented in 3D plots. The RSM generated 3D plots are used to analyze the effect of the interactions of the variables, and the plots are generated with response on the $z$-axis against two independent variables with third variable kept constant (Figs. 1, 2, 3, 4, 5).

\section{Effect of the independent variables interactions on the production of bioactive metabolite and its antagonistic effect on the responses}

Effect of the different variables with linear square and quadratic coefficients was obtained at significant terms. As single, the factors like concentration of sucrose and soya peptone had high coefficient value which showed their high linear significant effect on growth inhibition of pathogenic microorganisms by the bioactive compound produced by the strain. Direct effect of the five variables, time of incubation, $\mathrm{pH}$, temperature, concentration of sucrose, and concentration of soya peptone on the growth inhibition of the five response pathogens by the bioactive metabolites produced by the strain, is represented by zone of inhibition (mm). The stationary phase of the strain $N$. litoralis strain VSM 8 extended from 5 th to 11 th day. The maximum yield of the bioactive metabolite was recorded on the 11 day.

Venkata et al. (2011) reported that Amycolatopsis alba var. nov. DVR D4 strain produced maximum bioactive compound after 4 days of incubation. However, AbdElnaby et al. (2016) reported the maximum production of the bioactive metabolites by Streptomyces parvus was obtained after 7 days of incubation. The effect of the environmental factors, such as temperature and $\mathrm{pH}$ on the bioactive metabolite production, was studied. When the temperature varied from 20 to $30^{\circ} \mathrm{C}$, the yield of the bioactive metabolite increased gradually and the maximum production was recorded at $25{ }^{\circ} \mathrm{C}$. There was a decrease in the production of the bioactive metabolite with further increase in temperature. Joseph et al. (2009) reported maximum inhibitory activity of the bioactive metabolite produced by marine Nocardiopsis dassonvillei MAD08 cultured at $30{ }^{\circ} \mathrm{C}$. Krishna Kumar et al. (2011) also reported the optimum temperature for the bioactive metabolites production by Streptomyces sp.-MSU29 as $30{ }^{\circ} \mathrm{C}$. Venkata et al. (2011) reported that the ideal temperature for the production of the maximum bioactive metabolite by Amycolatopsis alba var. nov. DVR D4 was $28{ }^{\circ} \mathrm{C}$. Kerstin et al. (2010) reported the marine strain Nocardiopsis produced high levels of Thiopeptide Antibiotic, TP-1161 after 14 days of incubation.

The effect of the environmental factors, such as temperature and $\mathrm{pH}$ on growth and bioactive metabolite production, was studied. Varied temperatures between 20 and $30{ }^{\circ} \mathrm{C}$ were tested for the production of the bioactive compound by $N$. litoralis strain VSM 8 . The maximum production of the bioactive metabolite was recorded at $25{ }^{\circ} \mathrm{C}$. These results are in accordance with the results reported by Attiya et al. (2015) who reported the optimum temperature for the production of the bioactive compound by marine Streptomyces spp. M19 was $25^{\circ} \mathrm{C}$. Vimal et al. (2009) have reported that the maximum activity of the bioactive metabolite produced by Nocardiopsis sp. VITSVK 5 was recorded at $28{ }^{\circ} \mathrm{C}$.

Maximum growth and the elevated level of bioactive metabolite production by $N$. litoralis strain VSM 8 was found to be at $\mathrm{pH} 8$. Increase in $\mathrm{pH}$ resulted in the decreased production of the bioactive metabolite. Similar results were reported by Abd-Elnaby et al. (2016) for Streptomyces parvus. Kerstin et al. (2010) reported the optimum $\mathrm{pH}$ for the production of thiopeptide Antibiotic, TP-1161 by marine strain Nocardiopsis was 7.8. Designing and developing the effective media for the bioactive metabolite production are critical, and the impact of carbon needs to be evaluated. Of all the carbon sources tested, significant production of the bioactive metabolites by $N$. litoralis strain VSM 8 was found when the medium was 
Fig. 1 Response surface plots consisting of three-dimensional views and contours showing interactive effects of selective variables on zone of inhibition $(\mathrm{mm})$ of the bioactive compound production by VSM 8 against Staphylococcus aureus: $(A B)$ time of incubation and $\mathrm{pH},(A C)$ time of incubation and temperature, $(A D)$ time of incubation and concentration of sucrose, $(A E)$ time of incubation and concentration of soya peptone, $(B C) \mathrm{pH}$ and temperature, $(B D) \mathrm{pH}$ and concentration of sucrose, $(B E)$ $\mathrm{pH}$ and concentration of soya peptone, $(C D)$ temperature and concentration of sucrose, $(C E)$ temperature and concentration of soya peptone, $(D E)$ concentration of sucrose and concentration of soya peptone
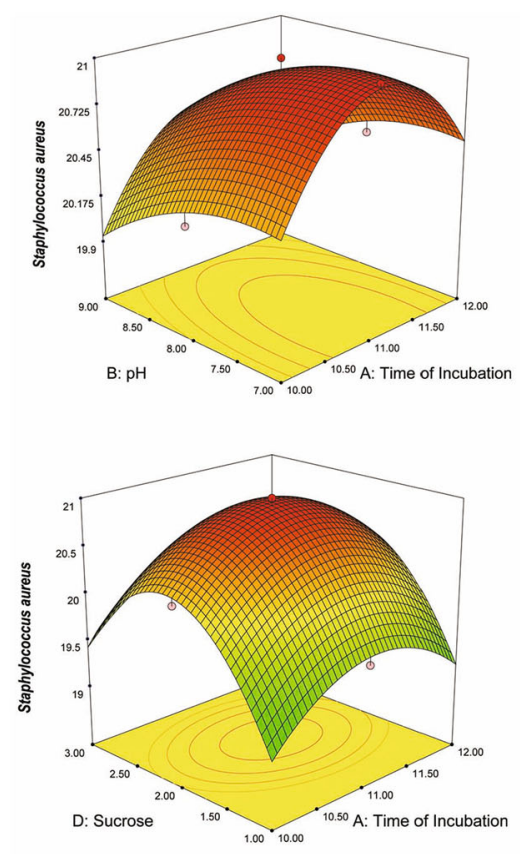

$\mathrm{AB}$
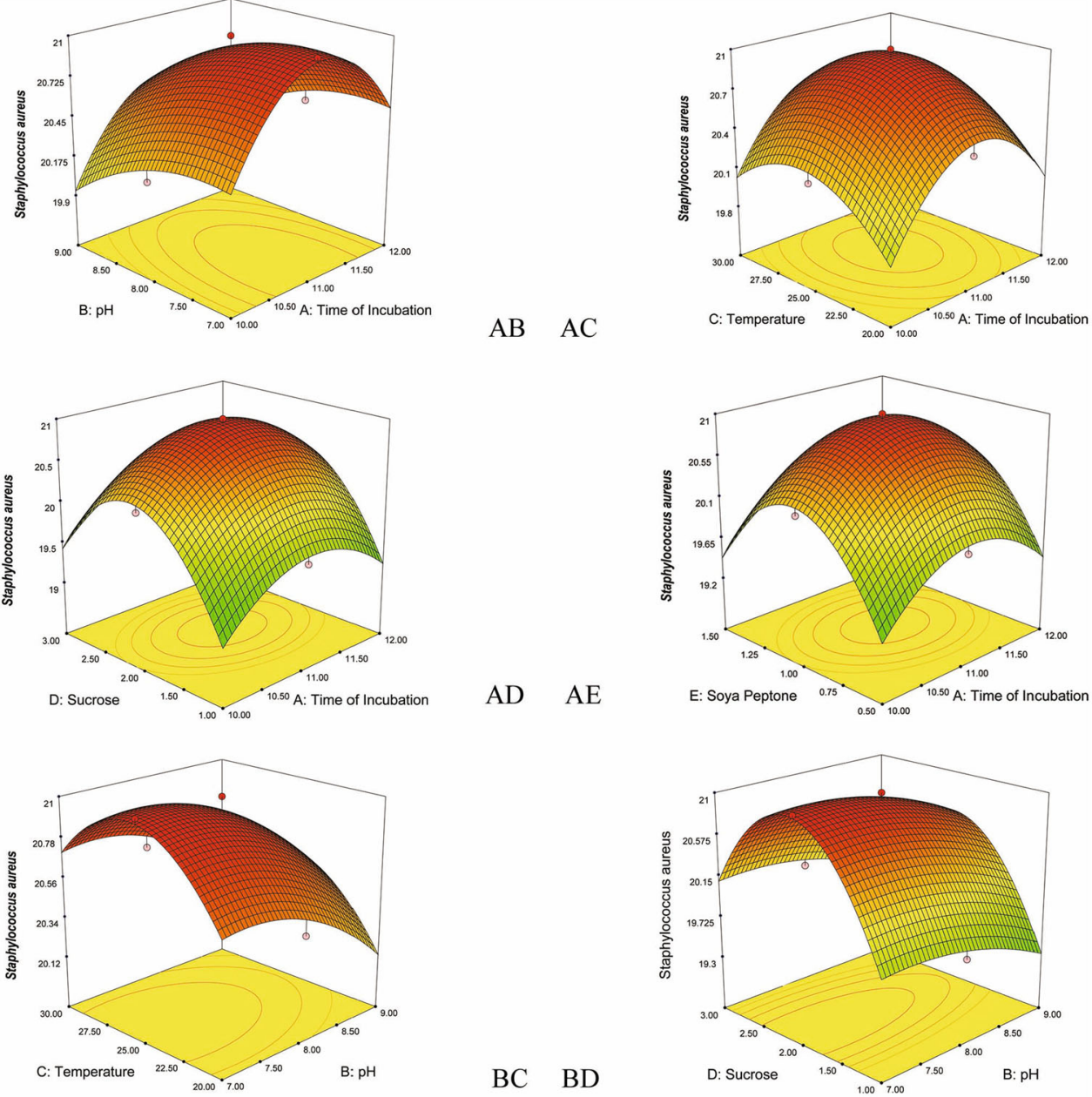

$\mathrm{AD}$

AE
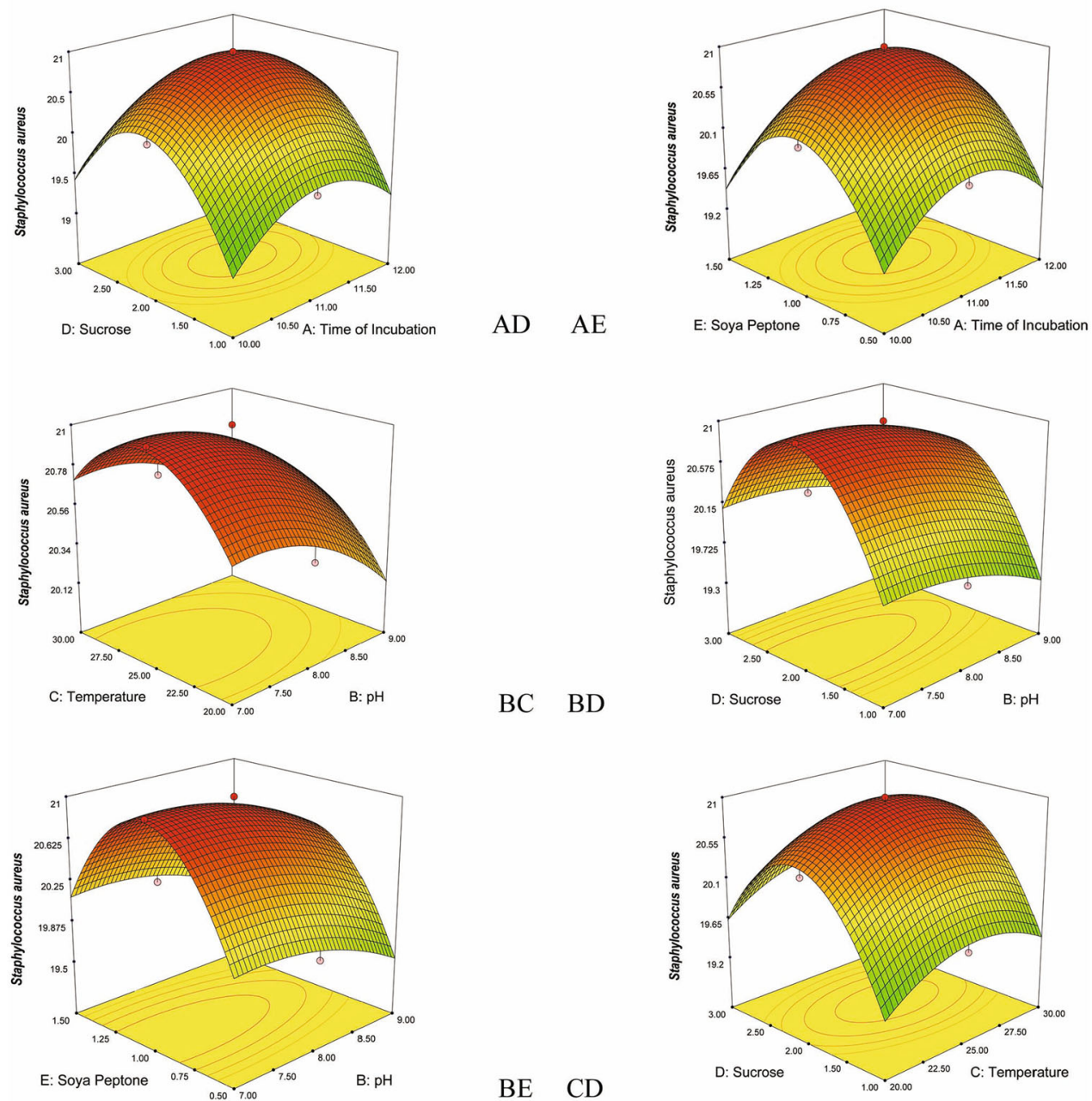

$\mathrm{BC}$

$\mathrm{BD}$
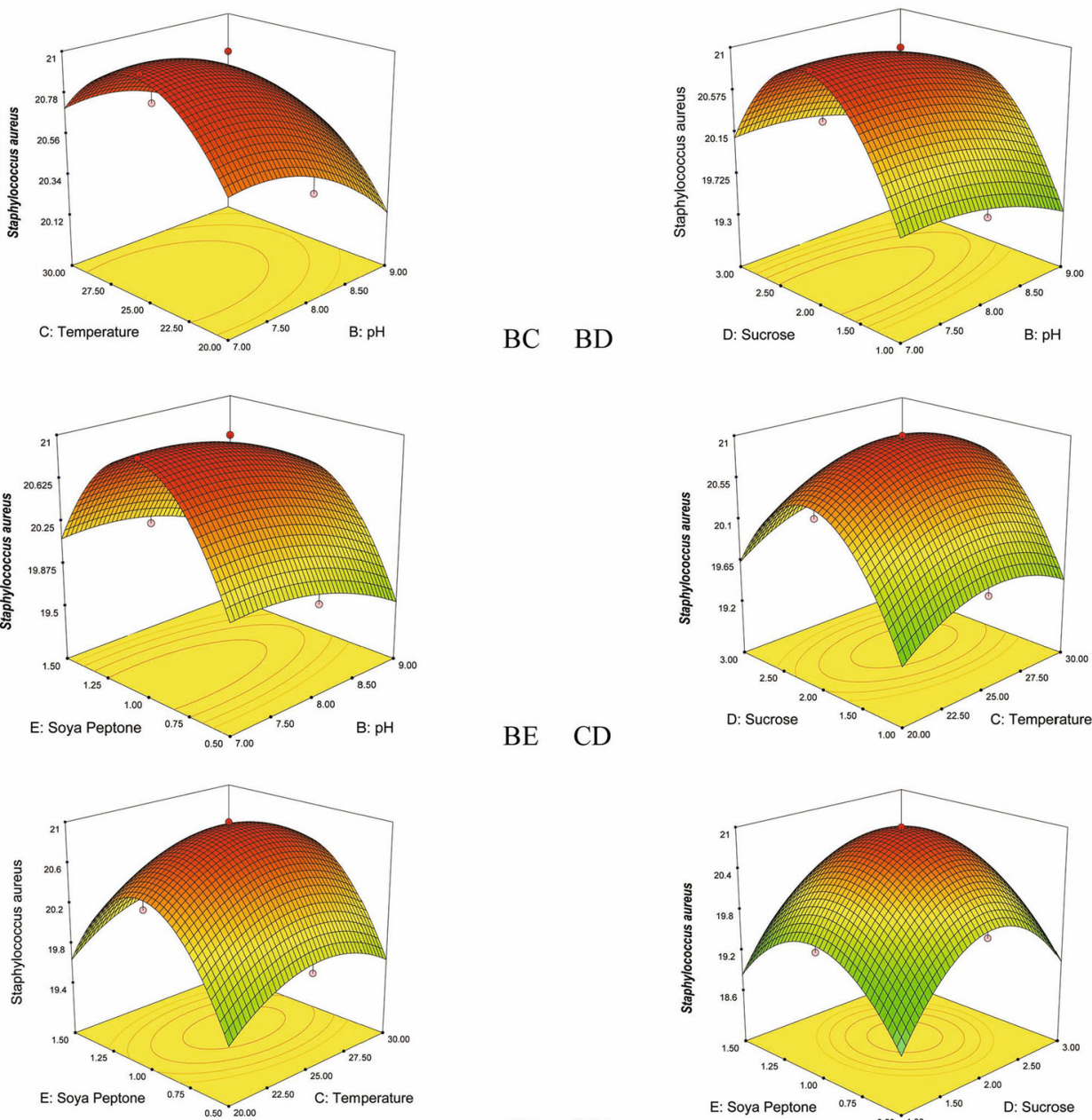

BE CD
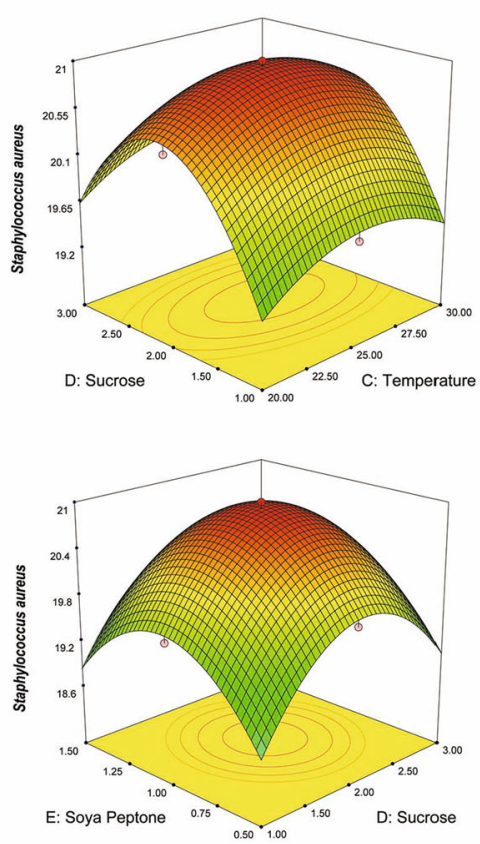
Fig. 2 Response surface plots consisting three-dimensional views and contours showing interactive effects of selective variables on zone of inhibition (mm) of the bioactive compound production by VSM 8 against Bacillus subtilis: $(A B)$ time of incubation and $\mathrm{pH},(A C)$ time of incubation and temperature, $(A D)$ time of incubation and concentration of sucrose, $(A E)$ time of incubation and concentration of soya peptone, $(B C) \mathrm{pH}$ and temperature, $(B D) \mathrm{pH}$ and concentration of sucrose, $(B E)$ $\mathrm{pH}$ and concentration of soya peptone, $(C D)$ temperature and concentration of sucrose, $(C E)$ temperature and concentration of soya peptone, $(D E)$ concentration of sucrose and concentration of soya peptone
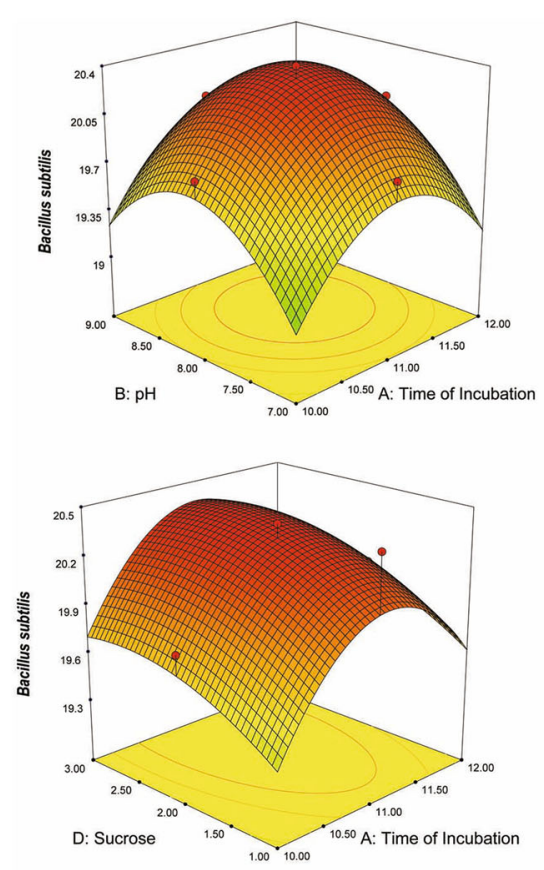

$\mathrm{AD}$

$\mathrm{AE}$
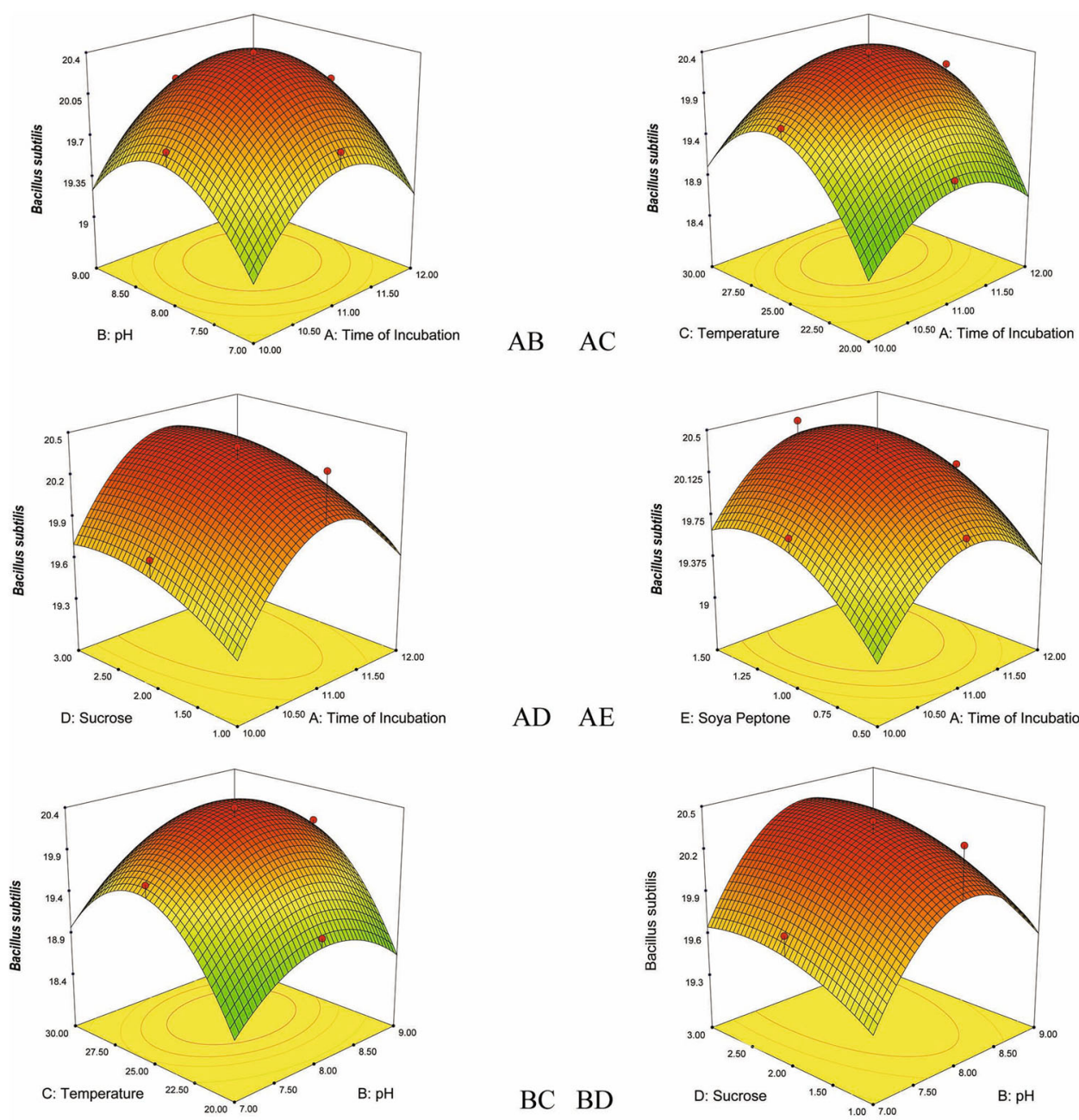

BC BD
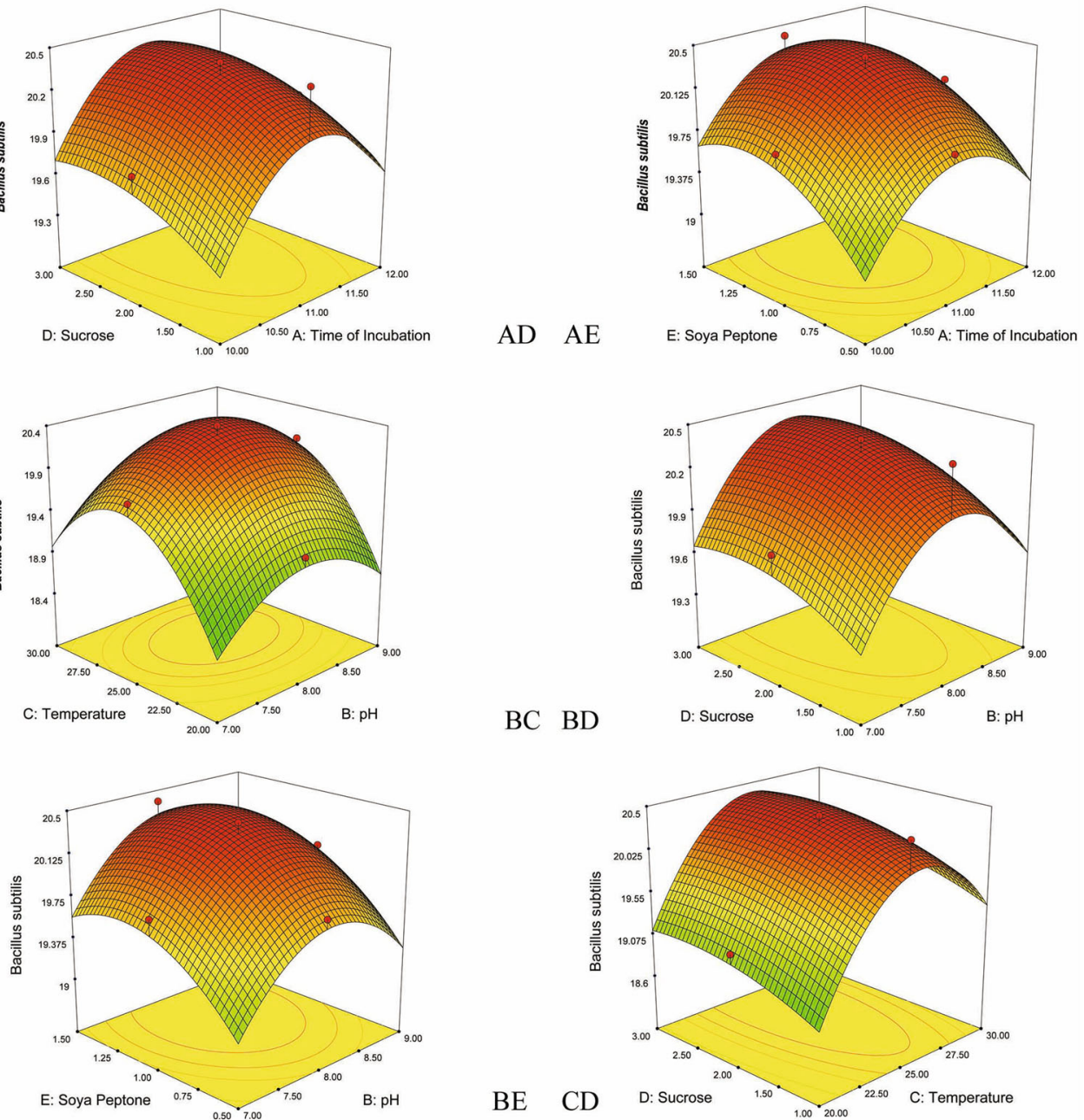

BE
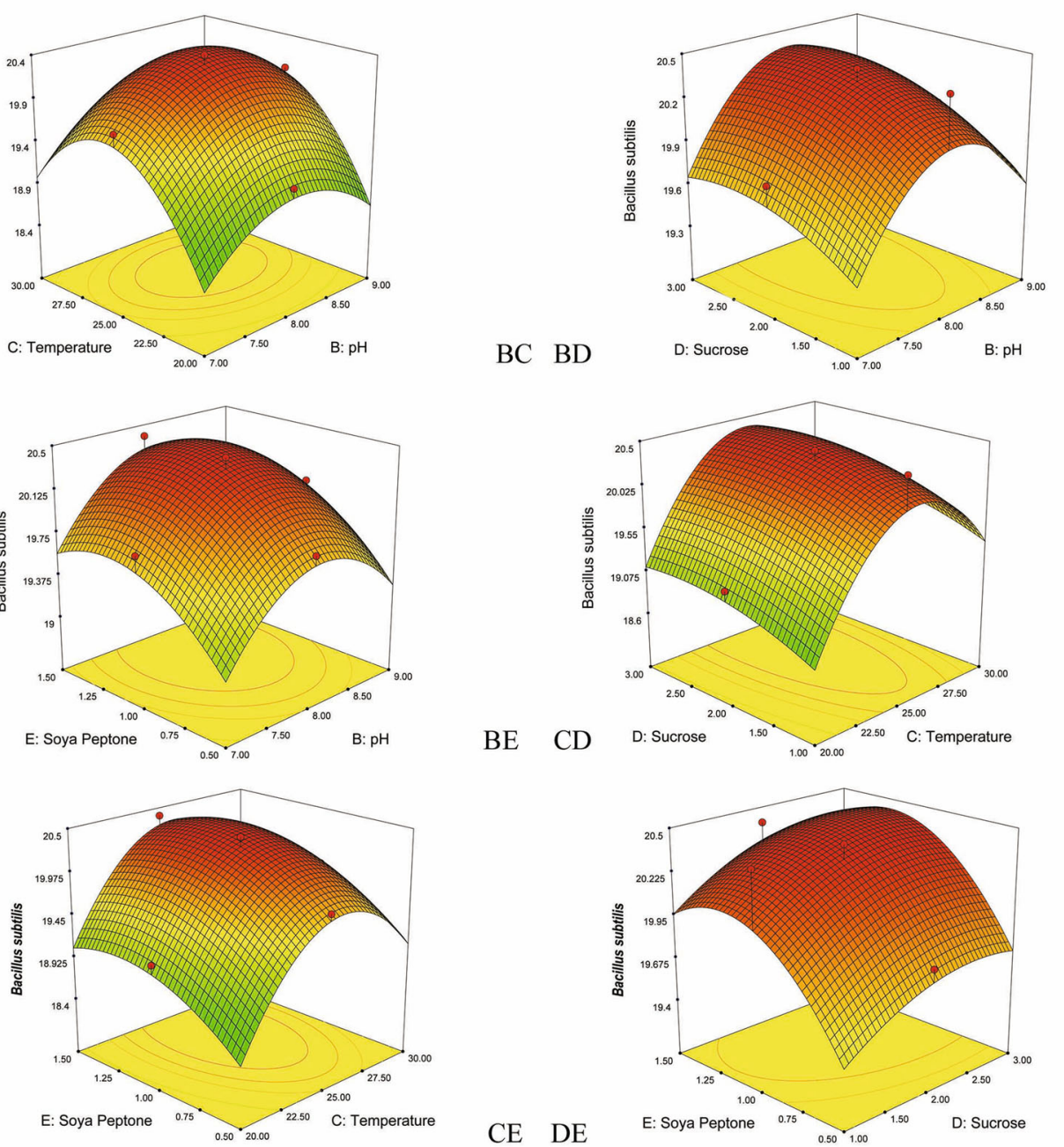

CE DE 
Fig. 3 Response surface plots consisting three-dimensional views and contours showing interactive effects of selective variables on zone of inhibition $(\mathrm{mm})$ of the bioactive compound production by VSM 8 against Xanthomonas campestris: $(A B)$ time of incubation and $\mathrm{pH}(A C)$ time of incubation and temperature $(A D)$ time of incubation and concentration of sucrose, $(A E)$ time of incubation and concentration of soya peptone, $(B C) \mathrm{pH}$ and temperature, $(B D) \mathrm{pH}$ and concentration of sucrose, $(B E)$ $\mathrm{pH}$ and concentration of soya peptone, $(C D)$ temperature and concentration of sucrose, $(C E)$ temperature and concentration of soya peptone, $(D E)$ concentration of sucrose and concentration of soya peptone
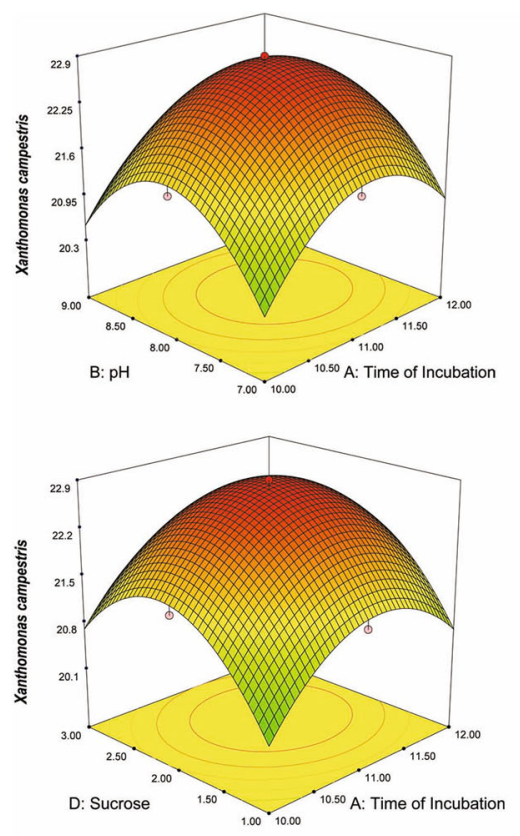

AB AC
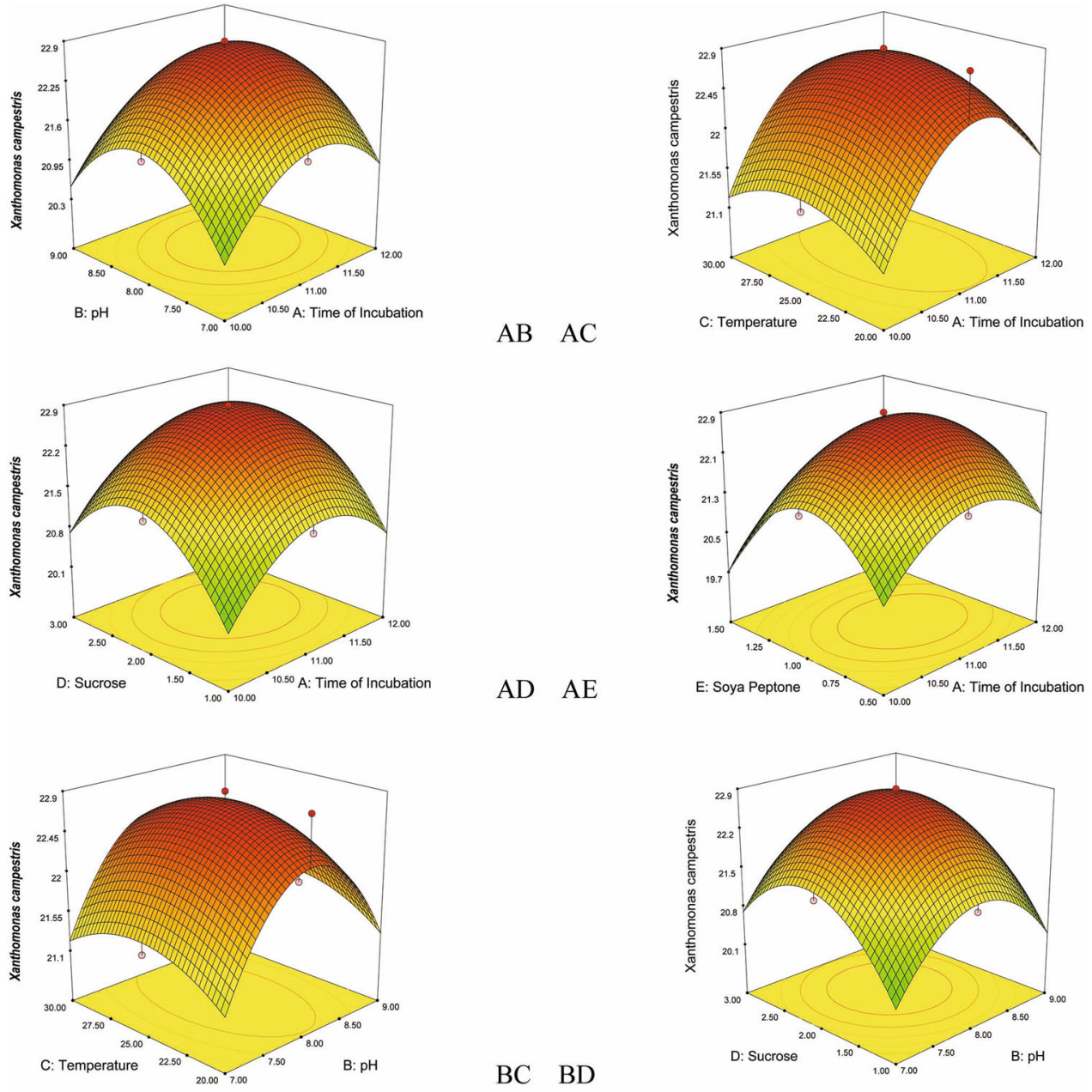

$\mathrm{AD} \quad \mathrm{AE}$
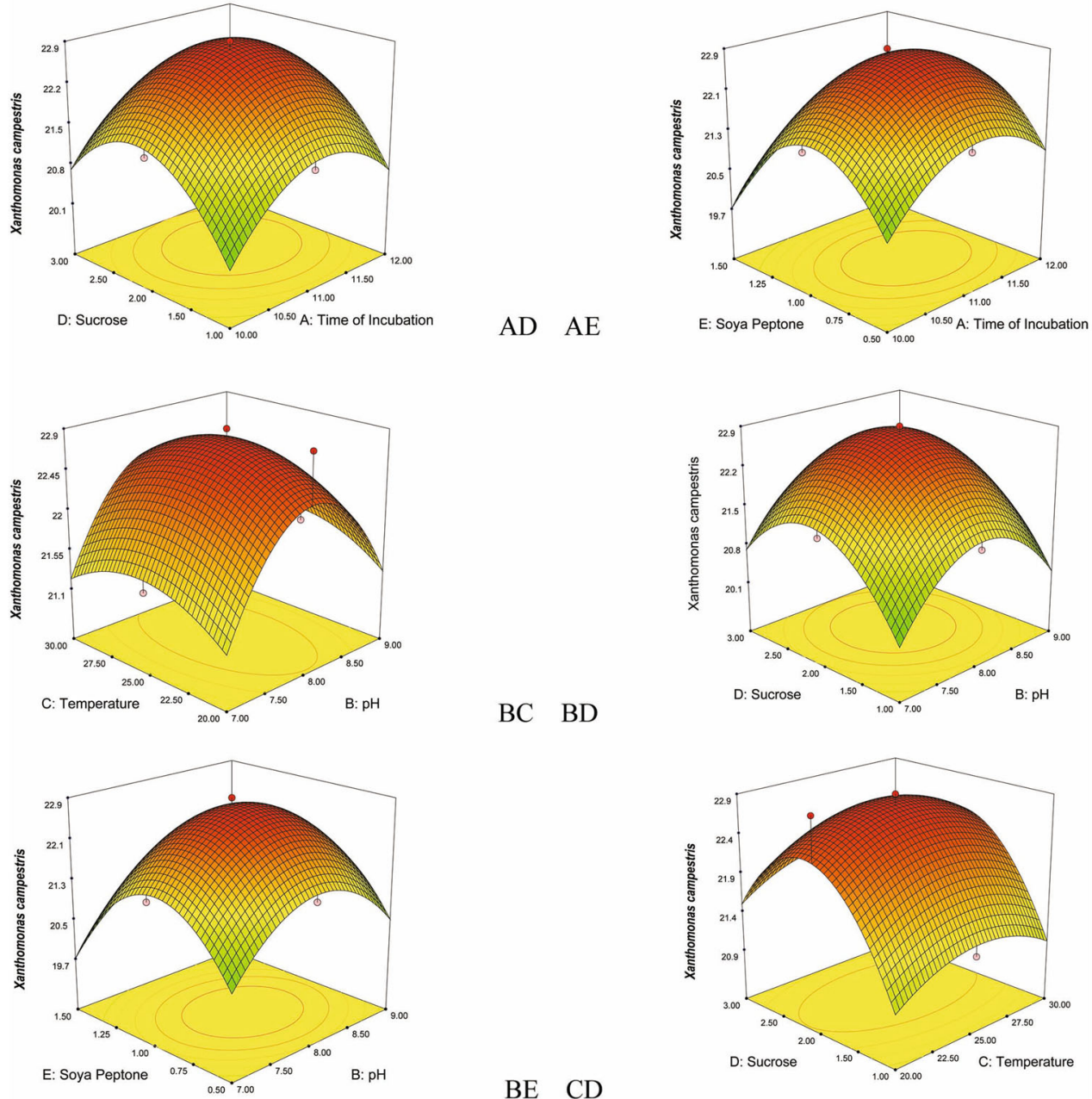

BC BD
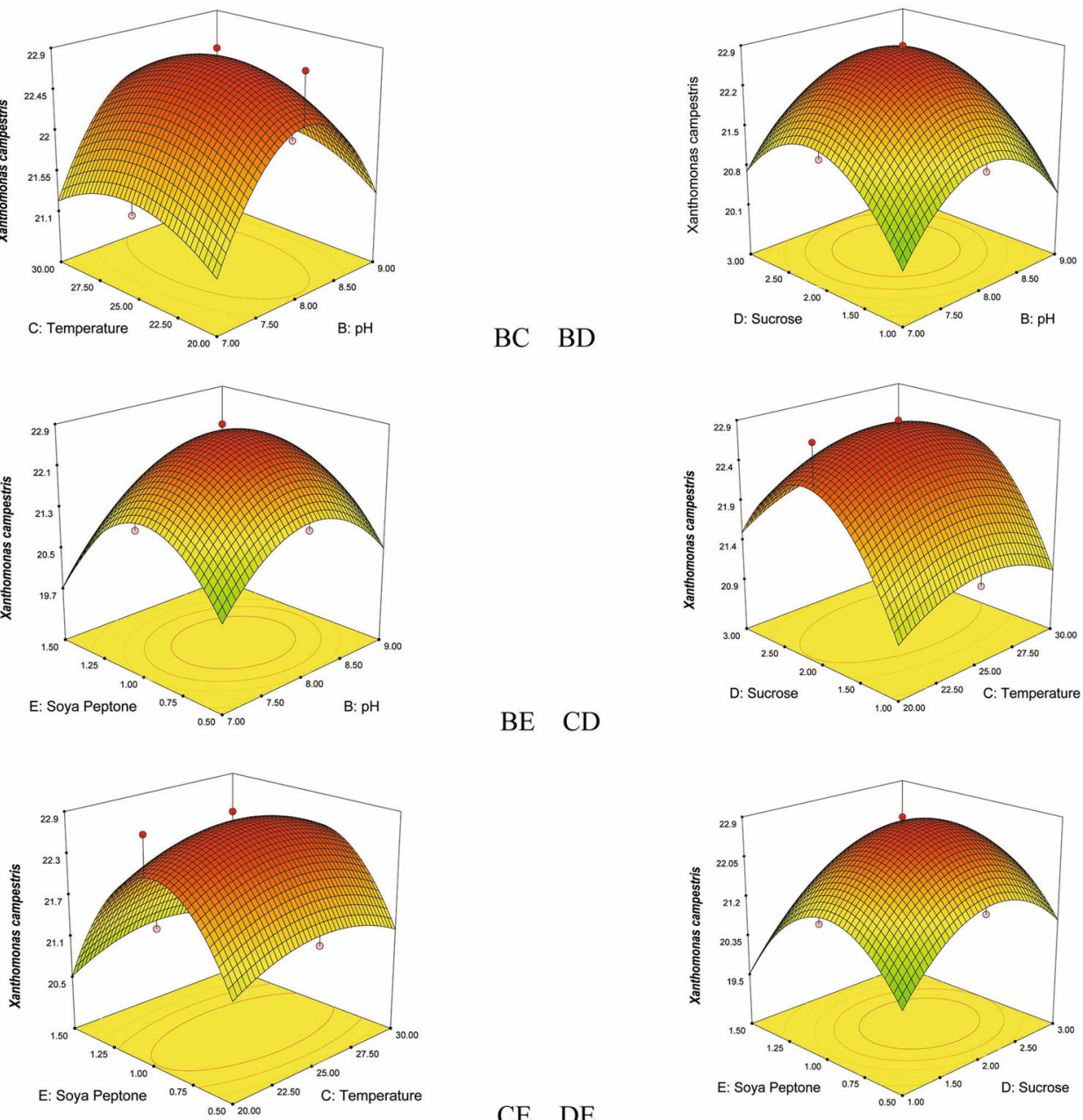

BE CD

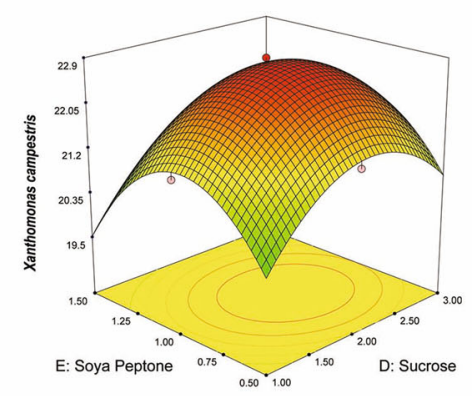

CE DE

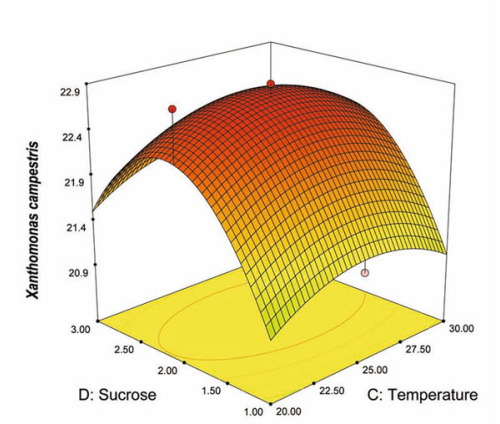

$\checkmark$

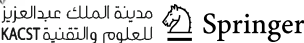


Fig. 4 Response surface plots consisting three-dimensional views and contours showing interactive effects of selective variables on zone of inhibition (mm) of the bioactive compound production by VSM 8 against Pseudomonas aeruginosa: $(A B)$ time of incubation and $\mathrm{pH},(A C)$ time of incubation and temperature, $(A D)$ time of incubation and concentration of sucrose, $(A E)$ time of incubation and concentration of soya peptone, $(B C) \mathrm{pH}$ and temperature, $(B D) \mathrm{pH}$ and concentration of sucrose, $(B E)$ $\mathrm{pH}$ and concentration of soya peptone, $(C D)$ temperature and concentration of sucrose, $(C E)$ temperature and concentration of soya peptone, $(D E)$ concentration of sucrose and concentration of soya peptone
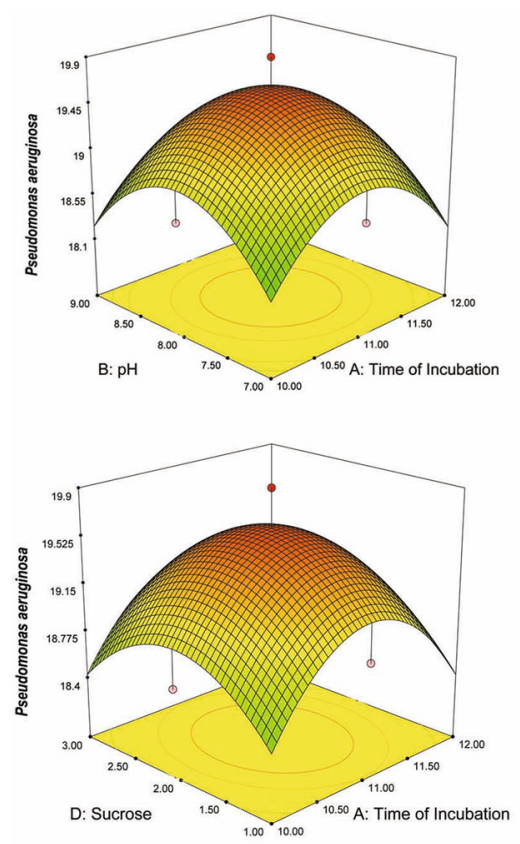

$\mathrm{AB}$

$\mathrm{AC}$
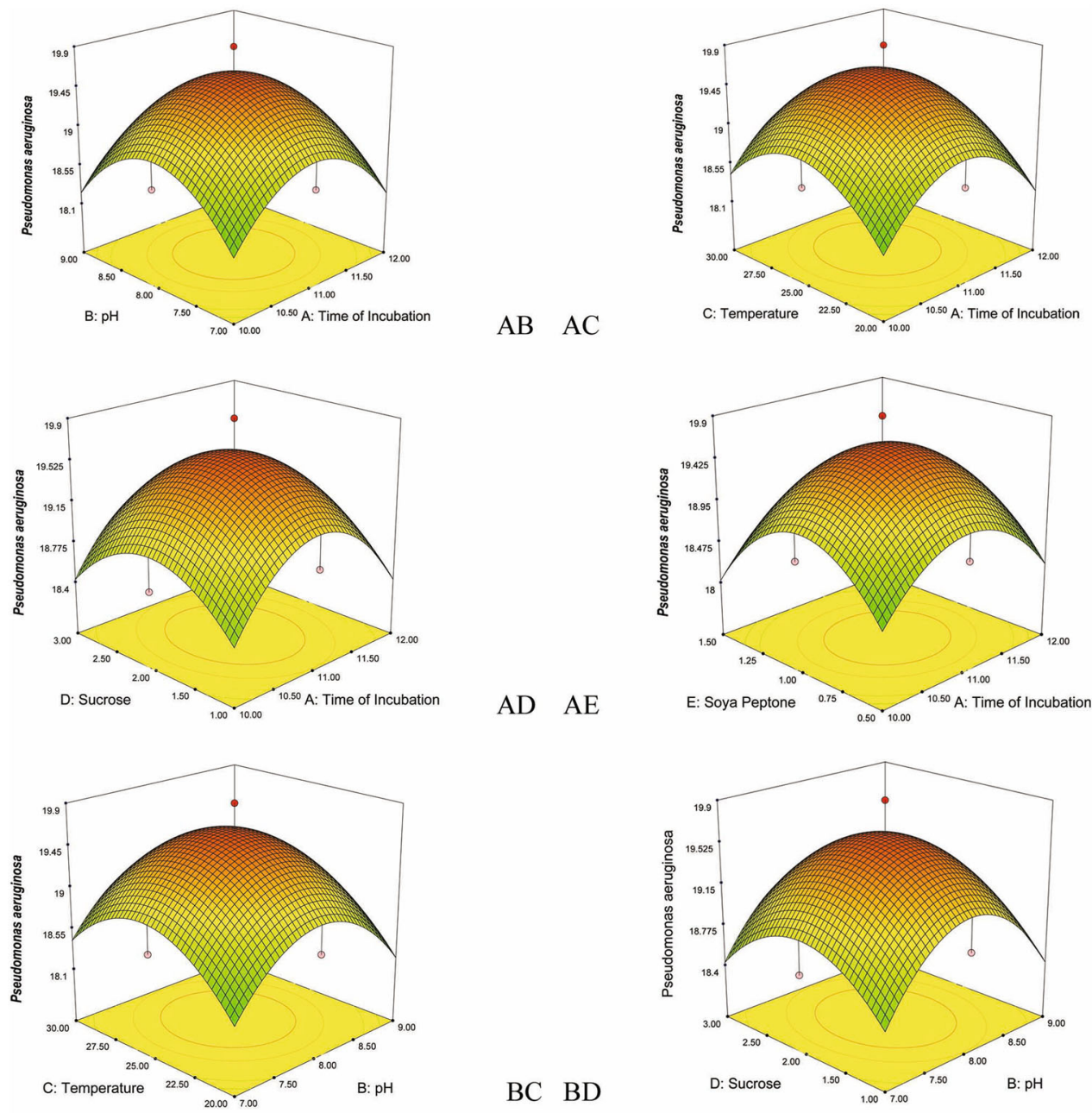

$\mathrm{AD}$

$\mathrm{AE}$
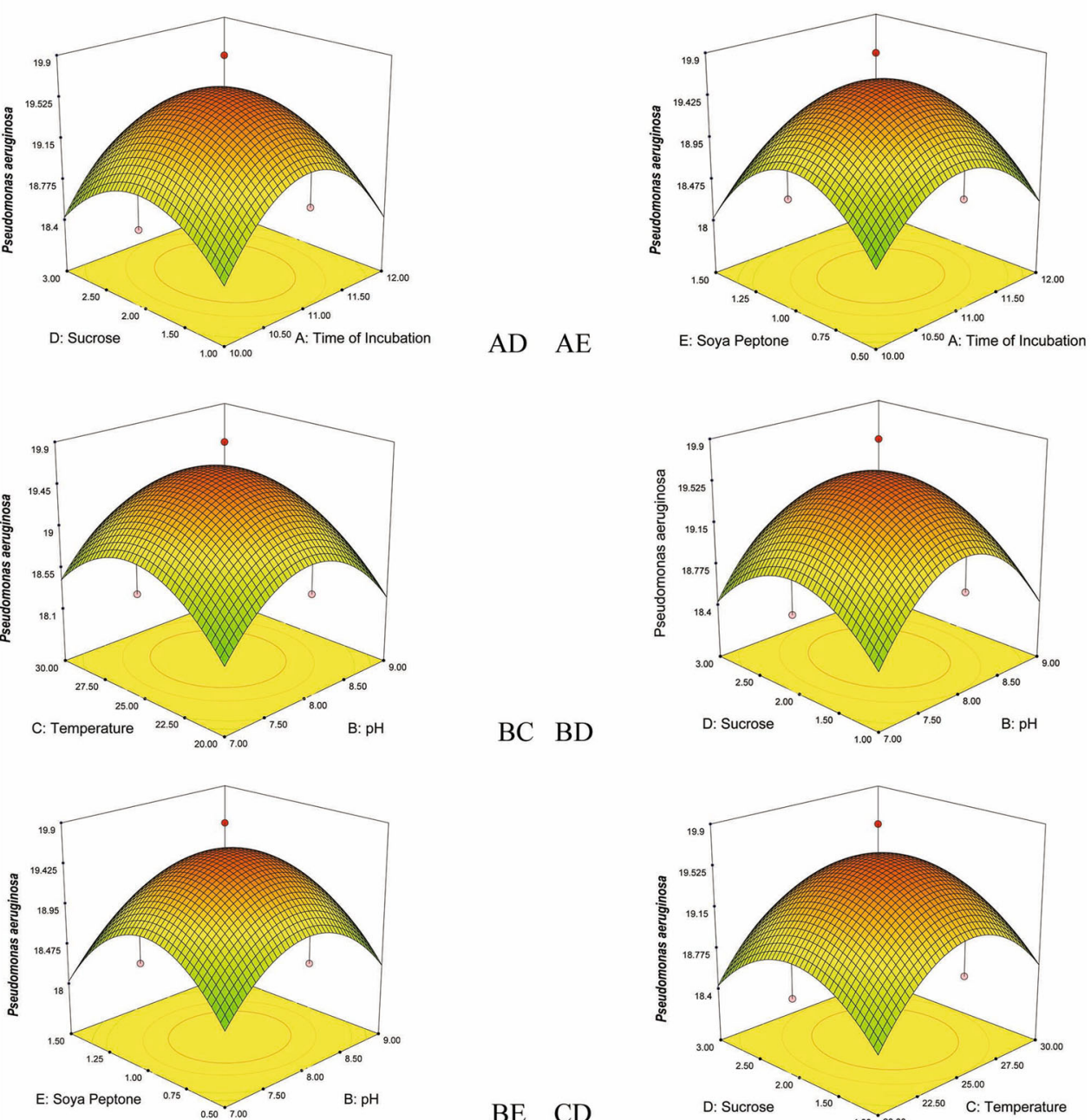

BE CD


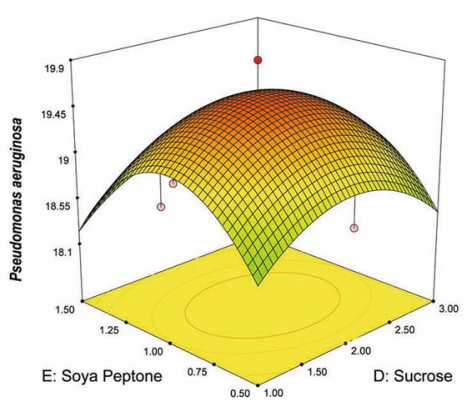

CE DE 
Fig. 5 Response surface plots consisting three-dimensional views and contours showing interactive effects of selective variables on zone of inhibition $(\mathrm{mm})$ of the bioactive compound production by VSM 8 against Candida albicans: $(A B)$ time of incubation and $\mathrm{pH},(A C)$ time of incubation and temperature, $(A D)$ time of incubation and concentration of sucrose, $(A E)$ Time of incubation and concentration of soya peptone, $(B C) \mathrm{pH}$ and temperature, $(B D) \mathrm{pH}$ and concentration of sucrose, $(B E)$ $\mathrm{pH}$ and concentration of soya peptone, $(C D)$ temperature and concentration of sucrose, $(C E)$ temperature and concentration of soya peptone, $(D E)$ concentration of sucrose and concentration of soya peptone
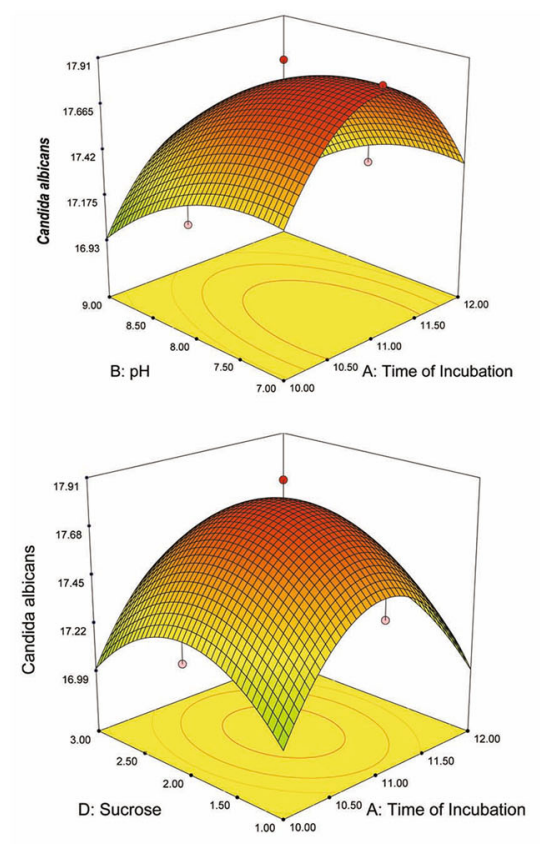

AB AC
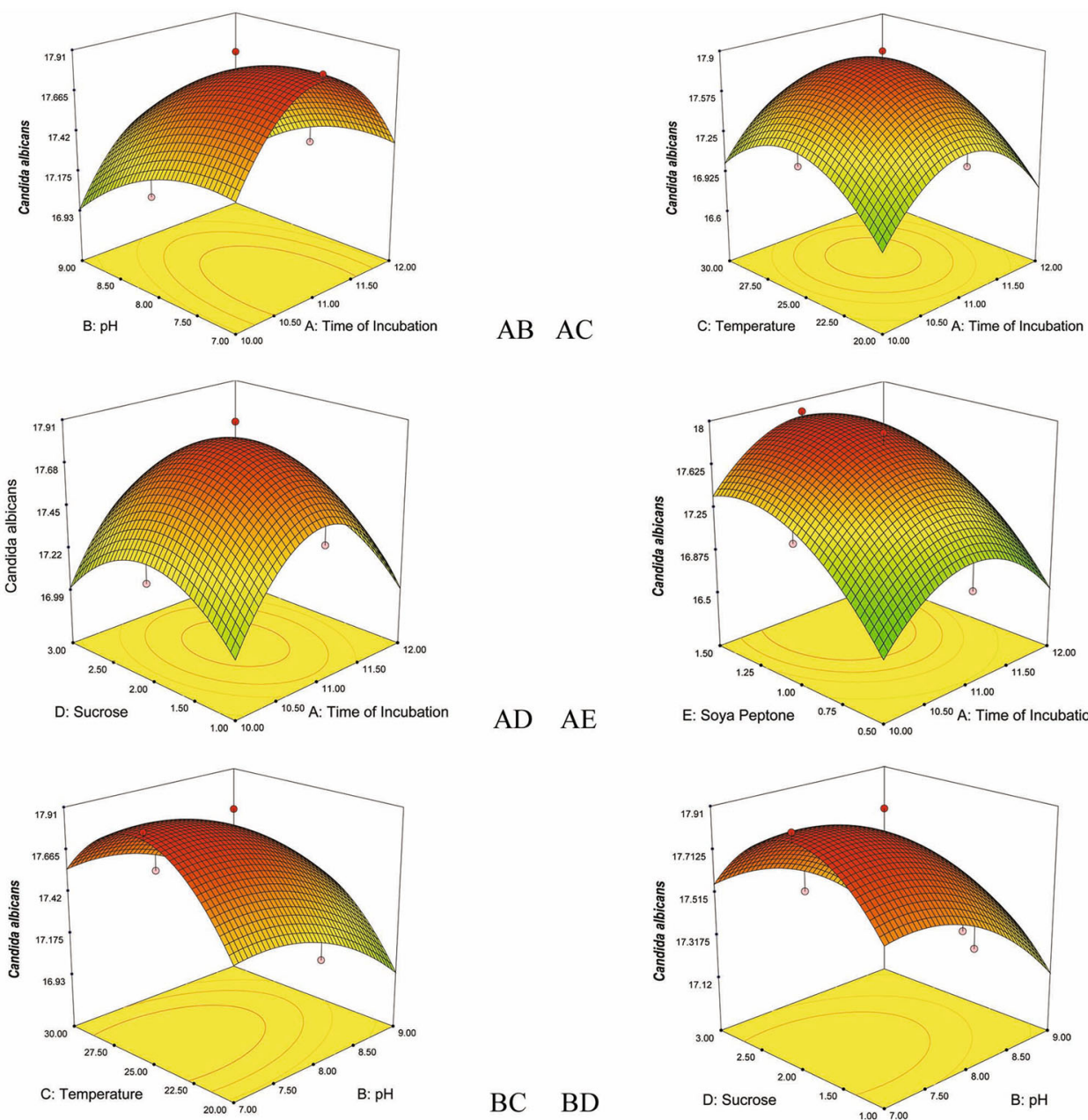

AD $\mathrm{AE}$
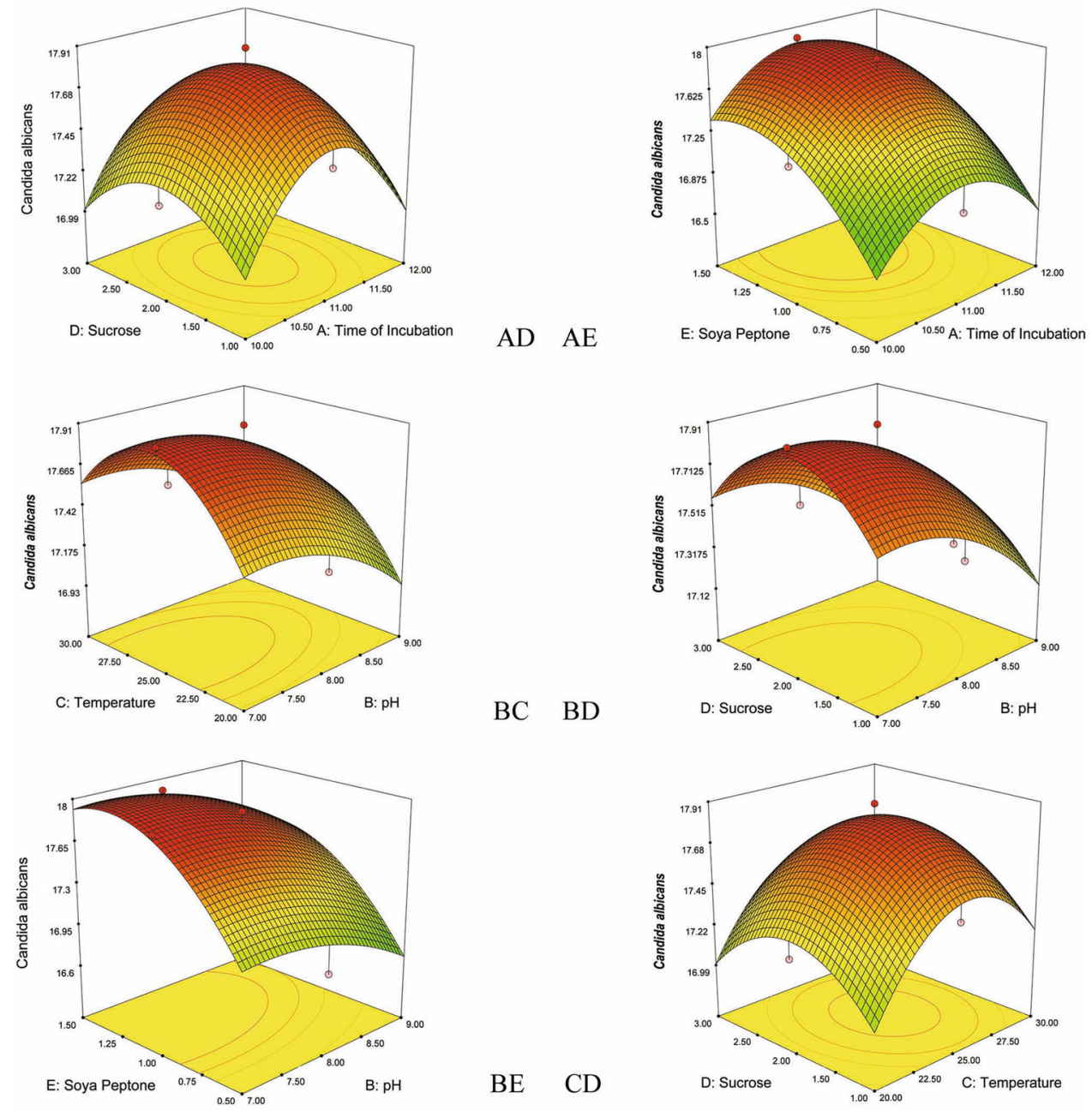

BC

BD
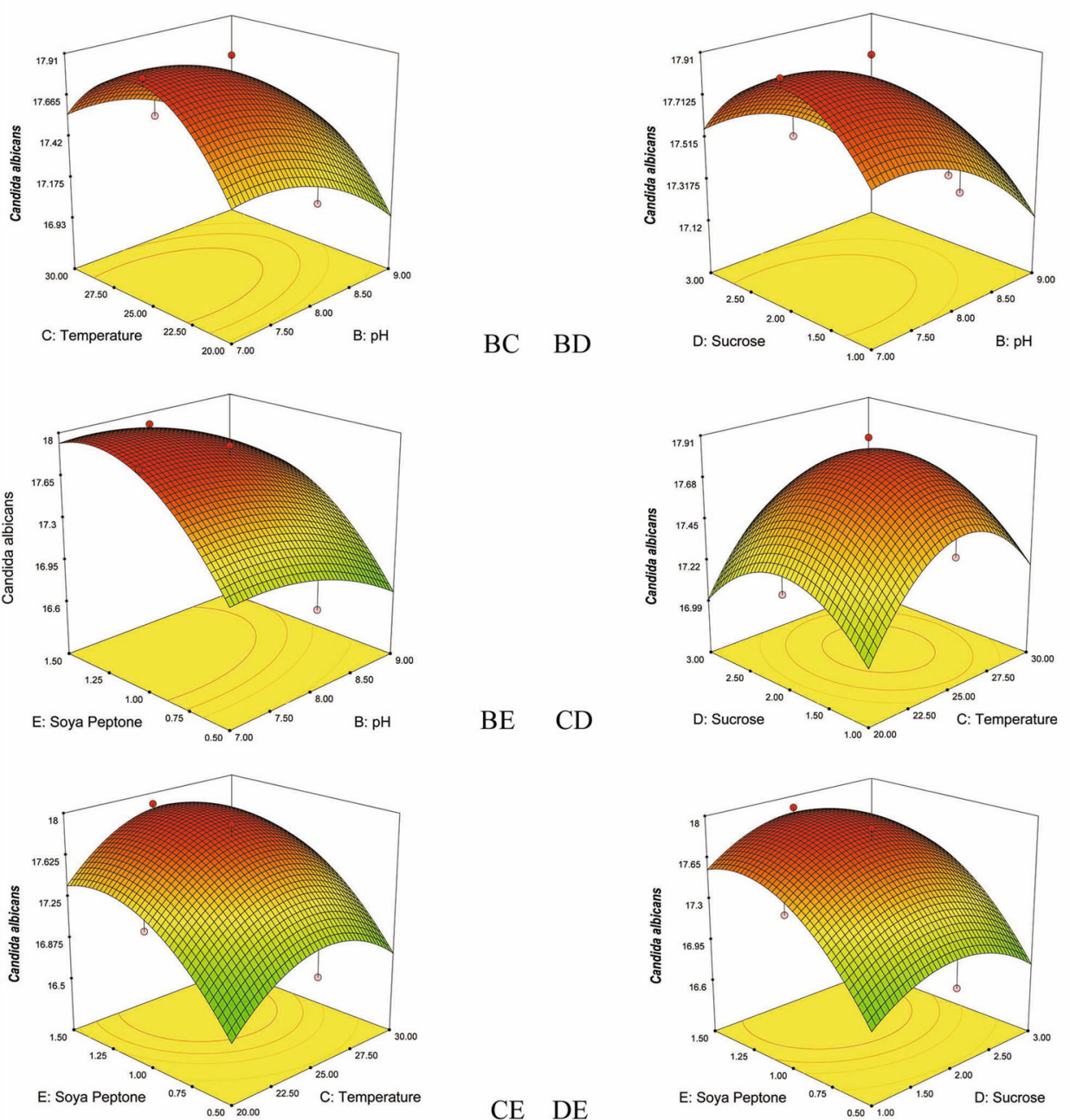

CE DE
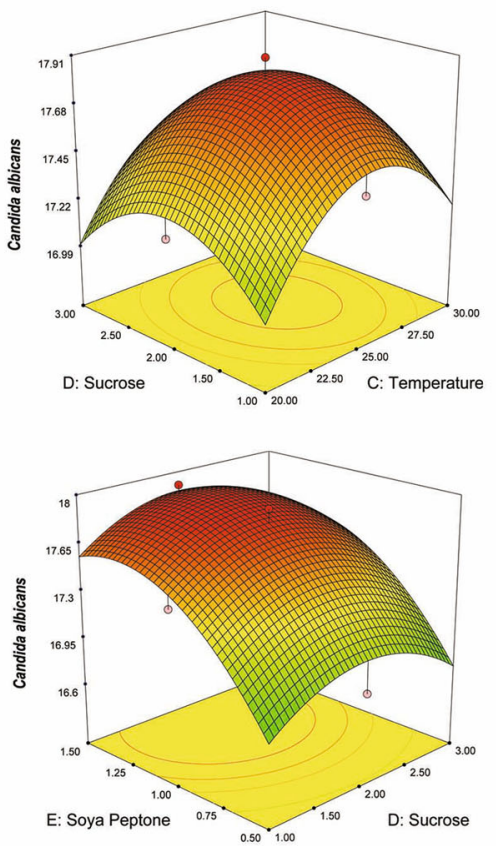
amended with sucrose. The effect of varying concentrations (1-3\%) of the best carbon source selected for the growth and antimicrobial metabolite production was also investigated. The result is in conformity with the results reported by Uddin et al. (2013) for Streptomyces albolongus, Streptomyces spp. KGG32 (Oskay 2011) and Streptomyces rochei G-164 (Chattopadhyay and Sen 1997).

Among the nitrogen sources tested, soya peptone at concentration $1 \%$ was found to influence that maximum production of the bioactive metabolite by $N$. litoralis strain VSM 8. The utilization of the nitrogen source for the production of bioactive metabolites is reported to vary for actinomycetes strains. Singh et al. (2009) recorded that

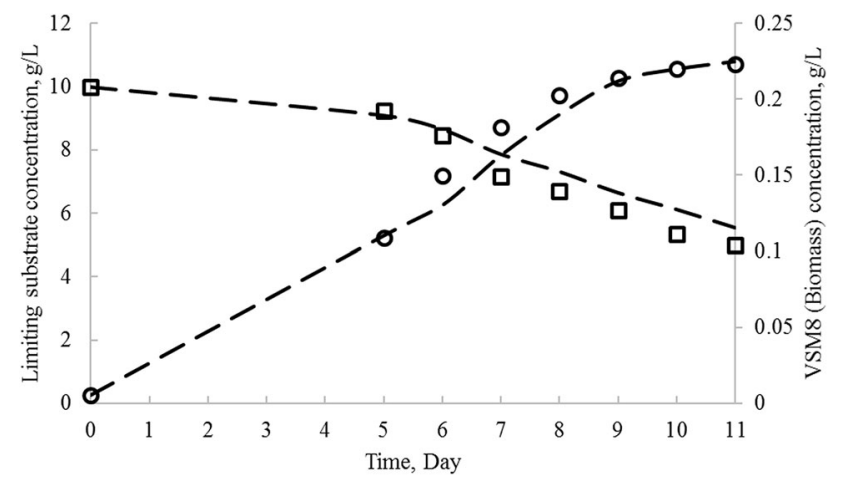

Fig. 6 Experimental and model predicted kinetics of biomass growth, substrate utilization. Open circle-experimental biomass concentration $(\mathrm{g} / \mathrm{L})$, open square - experimental substrate concentration $(\mathrm{g} / \mathrm{L})$, dashed lines - model predicted values (in each case) soya bean meal increased antibiotic production by Streptomyces tanashiensis strain $\mathrm{A} 2 \mathrm{D}$ and similar results were recorded by Narayana and Vijayalakshmi (2008), for Streptomyces albidoflavus.

\section{Discussion}

RSM, a collection of statistical and mathematical method, is used in conjunction with central composite design to optimize the different variables at different levels for the production of bioactive metabolite by $N$. litoralis strain VSM 8. Five variables include incubation time, $\mathrm{pH}$, temperature, concentration of sucrose, and soya peptone was optimized by central composite design involving RSM. A high similarity was observed between the predicted and the observed results that reflect the accuracy and accountability of the RSM to optimize the bioactive metabolites production. Of the five variables tested for the correlation between their concentration and production of the bioactive metabolite and its effect against the five responses, all the five variables exhibited significant effect on the production of the bioactive metabolite and their effect against the five pathogens (responses) which is represented as zone of inhibition. Significant interactions between the five variables were observed and analyzed from the 3D surface plots. Application of RSM with CCD statistical experimental design to optimize the selected factors for maximum production is an efficient method that tests the effect
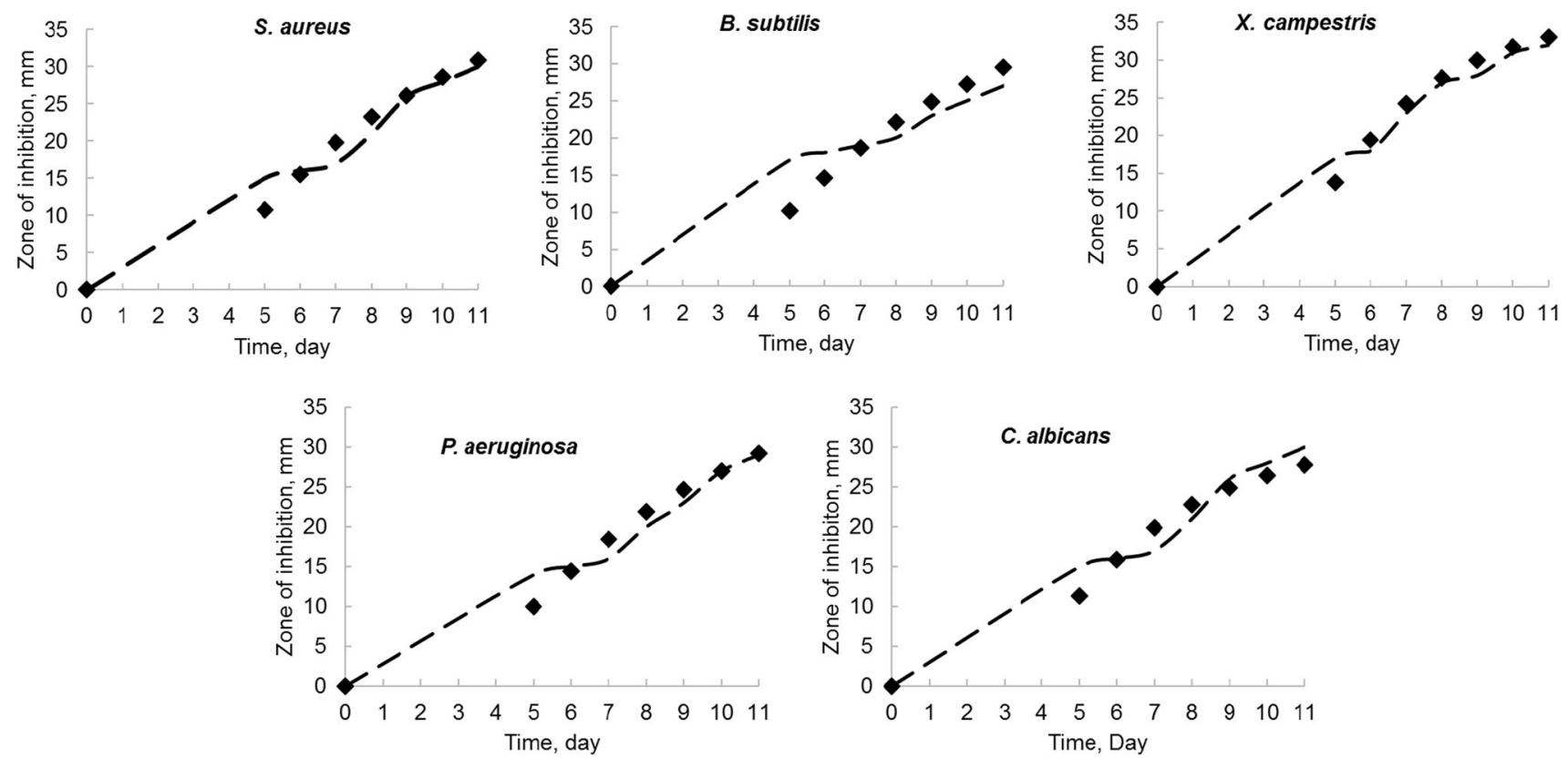

Fig. 7 Comparison of experimental and model predicted kinetics of zone of inhibition (mm). Filled diamond-experimental, dashed linesmodel 
Table 10 Estimated kinetic parameters using L, LILP, LIMLP model equations

\begin{tabular}{|c|c|c|c|c|c|}
\hline Kinetic Parameters & S. aureus & B. subtilis & X. campestris & P. aeruginosa & C. albicans \\
\hline \multicolumn{6}{|c|}{ Logistic (L) model parameters } \\
\hline$\mu_{\max }\left(\right.$ day $\left.^{-1}\right)$ & 0.7431 & & & & \\
\hline$R^{2}$ & 0.9683 & & & & \\
\hline$X_{0}(\mathrm{~g} / \mathrm{L})$ & 0.005 & & & & \\
\hline$X_{\max }(\mathrm{g} / \mathrm{L})$ & 0.226 & & & & \\
\hline \multicolumn{6}{|c|}{ Logistics incorporated modified Luedeking-Piret (LIMLP) model parameters } \\
\hline$\gamma($ g.S/g.X) & 4.8774 & & & & \\
\hline$R^{2}$ & 0.96 & & & & \\
\hline$\eta[$ g.S $/($ g.X.d) $]$ & 2.566371 & & & & \\
\hline \multicolumn{6}{|c|}{ Logistics incorporated Luedeking-Piret (LILP) model parameters } \\
\hline$\alpha(\mathrm{mm} / \mathrm{g} . \mathrm{X})$ & 87.224 & 81.246 & 124.62 & 79.93 & 100.24 \\
\hline$R^{2}$ & 0.9 & 0.54 & 0.9665 & 0.8877 & 0.9946 \\
\hline$\beta[\mathrm{mm} /(\mathrm{g} . \mathrm{X} . \mathrm{d})]$ & 8.8496 & 8.8496 & 4.4248 & 8.8496 & 4.4248 \\
\hline
\end{tabular}

Table 11 Comparison of zones of inhibition (mm) from shake-flask experiments and from model

\begin{tabular}{llllll}
\hline Maximum zone of inhibition $(\mathrm{mm})$ & S. aureus & B. subtilis & X. campestris & P. aeruginosa & C. albicans \\
\hline Experimental & 30 & 27 & 32 & 29 & 30 \\
Model fitted & 30.81 & 29.5 & 33.08 & 29.21 & 27.76 \\
\hline
\end{tabular}

of interactions among the variables with minimum number of experiments. Regression equations were derived for both selectivity and total flux using the experimental data together with the statistical software package Design Expert 7.1.3, yielding predicted values in good agreement with observed values.

The growth profiles of $N$. litoralis strain VSM 8 (KT901293), limiting substrate utilization results obtained from shake-flask experiments and model kinetics, were compared in Fig. 6. The comparison of experimental versus model predicted zones of inhibition of produced bioactive metabolite on media, inoculated with $S$. aureus, $B$. subtilis, $X$. campestris, $P$. aeruginosa, and $C$. albicans strains over the time, is shown in Fig. 7. From all the profiles, it was observed that model predicted and experimental obtained values show a very good fit. In this study, for fitting of experimental data with unstructured logistic models, non-linear regression using least-square method was done with the help of Microsoft Excel Solver 2010. Biokinetic parameters used in the mathematical model Eqs. (2), (4), and (7) were also estimated and are tabulated in Table 10. The determination coefficient $\left(R^{2}\right)$ values obtained by fitting logistic (L), logistic incorporated Leudeking-Piret (LILP), and logistic incorporated modified Leudeking-Piret (LIMLP) models to the experimental data were found to be high, thus revealing good precision of the models.
From the data of shake flask used in this study, $\mu_{\max }, X_{0}$ and $X_{\max }$ were calculated from $N$. litoralis strain VSM 8 (KT901293) growth kinetic profile using logistic (L) model. Values of growth and non-growth-associated product parameters, $\alpha$ and $\beta$, were estimated using LILP model. Higher $\alpha$ value than $\beta$ confirmed that bioactive metabolite production by $N$. litoralis strain VSM 8 is more growth-associated than non-growth associated in shake flask. The simulated parameters, $\gamma$ and $\eta$, of LIMLP model are also in good agreement with the experimental values, which implies that this model is more appropriate to represent limiting substrate utilization kinetics in bioactive metabolite production by $N$. litoralis strain VSM 8. Furthermore, zones of inhibition from agar diffusion tests are much similar to model predicted values (Table 11).

\section{Conclusions}

The effect of different variables, including incubation time, $\mathrm{pH}$, temperature, sucrose, and soya peptone concentration on production of bioactive metabolites by $N$. litoralis strain VSM 8 and the inhibiting activity of bioactive metabolites against five pathogens were studied in terms of their responses as inhibition zones $(\mathrm{mm})$. The experimental results showed that the maximum zone of inhibition against 
all the five responses was found to be $21 \mathrm{~mm}$ (Staphylococcus aureus), $19.8 \mathrm{~mm}$ (Bacillus subtilis), $22.9 \mathrm{~mm}$ (Xanthomonas campestris), $19.9 \mathrm{~mm}$ (Pseudomonas aeruginosa), and $17.9 \mathrm{~mm}$ (Candida albicans). The highest production of the bioactive metabolite produced by VSM 8 represented in terms of antimicrobial activity was obtained when the strain was grown in a medium containing $2 \%$ sucrose, $1 \%$ soya peptone with $\mathrm{pH} 8$ incubated at $25{ }^{\circ} \mathrm{C}$ for 11 days. However, the range of time of incubation, $\mathrm{pH}$, temperature, concentration of sucrose, and soya peptone above or below the central point reported less zone of inhibition.

A response surface experimental methodology, based on three levels central composite design of experiment, was successfully employed in this optimization study, accounting for the effects of the main variables. The quadratic models developed and subsequent ANOVA test, the concentration of sucrose and soya peptone dosage was found to be the most influential variables for the bioactive metabolite production along with the other significant variables. The model fitted very well to the experimental data, as confirmed by high $R^{2}$ values. RSM with CCD described the production of the bioactive compounds by $N$. litoralis strain VSM 8. Furthermore, the optimum conditions and the effect of bioactive compound against five responses are well induced by $3 \mathrm{D}$ plots.

The estimated kinetic parameters for the $N$. litoralis strain VSM 8 growth, and limiting substrate utilization and bioactive metabolite production (in terms of inhibition zones measured against microbial pathogens) showed good regression squares. Thus, the unstructured models provided a better approximation of kinetic profiles of bioactive metabolite production by $N$. litoralis strain VSM 8 in submerged shake-flask fermentations. To the best of our knowledge, this is the first ever report on the kinetic modeling for bioactive metabolite production measured in terms of zones of inhibition by $N$. litoralis strain VSM 8.

Acknowledgments This work was supported by the University grants commission, New Delhi, Government of India.

\section{Compliance with ethical standards}

Conflict of interest All the authors declare that there is no conflict of interest.

Open Access This article is distributed under the terms of the Creative Commons Attribution 4.0 International License (http:// creativecommons.org/licenses/by/4.0/), which permits unrestricted use, distribution, and reproduction in any medium, provided you give appropriate credit to the original author(s) and the source, provide a link to the Creative Commons license, and indicate if changes were made.

\section{References}

Abd-Elnaby H, Abo-Elala G, Abdel-Raouf U, Abd-elwahab A, Hamed M (2016) Antibacterial and anticancer activity of marine Streptomyces parvus: optimization and application. Biotechnol Biotechnol Equip 30:180-191

Attiya HM, Noura EE, AbdEl-Dayem AS, Suzan MH (2015) Optimization of bioactive metabolites production by a newly isolated marine Streptomyces sp. Using statistical approach. Biotechnol 14:211-224

Bipasha D, Ananda Prasad R, Sangita B, Sudip C, Chiranjib B (2015) Lactose hydrolysis by $\beta$-galactosidase enzyme: optimization using response surface methodology. Ecotoxicol Environ Saf 121:244-252

Chattopadhyay D, Sen SK (1997) Optimization of cultural conditions for antifungal antibiotic accumulation by Streptomyces rochei G164. Hindustan Antibiot Bull 39:64-71

Cheng KC, Demirci A, Catchmark JM, Puri VM (2010) Modeling of pullulan fermentation by using a color variant strain of Aureobasidium pullulans. J Food Eng 98:353-359

Collaa LM, Primaz AL, Benedetti S, Loss RA, de Limaa M, Reinehr CO, Bertolina TE, Costa JAV (2016) Surface response methodology for the optimization of lipase production under submerged fermentation by filamentous fungi. Braz J Microbiol 47:461-467

Cui P, Doua TY, Suna YP, Li SY, Fenga L, Zoua LW, Wanga P, Haoc DC, Gea GB, Yang L (2016) Efficient enzymatic preparation of esculentoside $\mathrm{B}$ following condition optimization by response surface methodology. J Mol Catal B Enzym 130:25-31

Ilaiyaraja N, Likhith KR, Sharath Babu GR, Farhath K (2015) Optimization of extraction of bioactive compounds from Feronia limonia (wood apple) fruit using response surface methodology (RSM). Food Chem 173:348-354

Joseph S, Shanmughapriya S, Gandhimathi R, Seghal Kiran G, Rajeetha Ravji T, Natarajaseenivasan K, Hema TA (2009) Optimization and production of novel antimicrobial agents from sponge associated marine actinomycetes Nocardiopsis dassonvillei MAD08. Appl Microbiol Biotechnol 83:435-445

Kerstin E, Kristin FD, Michael K, Harald B, Espen F, Geir K, Havard S, Trond EE, Sergey BZ (2010) Production of a new thiopeptide antibiotic, TP-1161, by a marine Nocardiopsis species. Appl Environ Microbiol 82:4969-4976

Kiranmayi MU, Sudhakar P, Sreenivasulu K, Vijayalakshmi M (2011) Optimization of culturing conditions for improved production of bioactive metabolites by Pseudonocardia spp. VUK-10. Mycobiology 39:174-181

Krishna Kumar S, Premkumar J, Alexis Rajan R, Ravi Kumar S (2011) Optimization of potential antibiotic production by salttolerant actinomycetes Streptomyces spp.-MSU29 isolated form marine sponge. Int J Appl Bio Eng 5:12-18

Leroy F, de Vuyst L (1999) Temperature and $\mathrm{pH}$ conditions that prevail during fermentation of sausages are optimal for production of the antilisterial bacteriocin sakacin K. Appl Environ Microbiol 65:974-981

Li HX, Lu ZM, Geng Y, Gong JS, Zhang XJ, Shi JS, Xu ZH, Ma YH (2015) Efficient production of bioactive metabolites from Antrodia camphorata ATCC 200183 by asexual reproduction-based repeated batch fermentation. Bioresour Technol 194:334-343

Luedeking R, Piret EL (2000) A kinetic study of the lactic acid fermentation. Batch process at controlled $\mathrm{pH}$. Biotechnol Bioeng 67:636-644

Mohammad FHA, Badr-Eldin SM, El-Tayeb OM, Abd El-Rahman OA (1995) Polysaccharide production by Aureobasidium pullulans III. The influence of initial sucrose concentration on batch kinetics. Biomass Bioenergy 8:121-129 
Narayana KJP, Vijayalakshmi M (2008) Optimization of antimicrobial metabolites production by Streptomyces albidoflavus. Res J Pharmacol 2:4-7

Oskay M (2011) Effects of some environmental conditions on biomass and antimicrobial metabolite production by Streptomyces sp., KGG32. Int J Agric Biol 13:317-324

Parmjit SP (2008) Application of response surface methodology in the permeabilization of yeast cells for lactose hydrolysis. Biochem Eng J 39:91-96

Pfefferle C, Theobald U, Gurtler H, Fiedler HP (2000) Improved secondary metabolite production in the genus Streptosporangium by optimization of the fermentation conditions. J Biotechnol 80:135-142

Rajendran A, Thangavelu V (2008) Evaluation of various unstructured kinetic models for the production of protease by Bacillus sphaericus MTCC 511. Eng Life Sci 8:179-185

Rama Krishna Ganduri VS, Ushakiranmayi M, Vijayalakshmi M, Sudhakar P (2016) Model-based kinetic parameters estimation in batch Pullulan fermentation using Jaggery as substrate. J Chem Pharma Res 8:217-224

Shekar P, Kumar KS, Jabasingh SA, Radhakrishnan M, Balagurunathan $\mathrm{R}$ (2014) Optimization of medium components for antibacterial metabolite production from marine Streptomycetes sp. Pua2 using response surface methodology. Int J Pharm Pharma Sci 6:475-480
Singh LS, Mazumdar S, Bora TC (2009) Optimization of process parameters for growth and bioactive metabolite production by a salt-tolerant and alkaliphilic actinomycete, Streptomyces tanashiensis strain A2D. J Mycol Med 19:223-225

Souagui Y, Tritsch D, Grosdemange-Billiard C, Kecha M (2015) Optimization of antifungal production by an alkaliphilic and halotolerant actinomycete, Streptomycetes sp. SY-BS5, using response surface methodology. J Mycol Med 25:108-115

Uddin M, Mahmud N, Anwar N, Manchur MA (2013) Bioactive metabolite production by Streptomyces albolongus in favourable environment. J Microbiol Infect Dis 3:75-82

Urailuck P, Weera P, Suthasinee T, Aran HK (2015) Enzymatic preparation of linoleic acid from sunflower oil: an experimental design approach. Biotechnol Biotechnol Equip 29:1-9

Venkata Ratna Ravi Kumar D, Murali Yugandhar N, Sri Rami Reddy D (2011) Screening of antagonistic marine actinomycetes: optimization of process parameters for the production of novel antibiotic by Amycolatopsis alba var. nov. DVR D4. J Microbial Biochem Technol 3:092-098

Vimal V, Benita Mercy R, Kannabiran K (2009) Antimicrobial activity of marine actinomycete, Nocardiopsis sp. VITSVK 5 (FJ973467). Asian J Med Sci 1:57-63 